\title{
Adult human neurogenesis: early studies clarify recent controversies and go further
}

Adriano Barreto Nogueira ${ }^{1,2^{*}}$, Hillary Sayuri Ramires Hoshino ${ }^{3}$, Natalia Camargo $\mathrm{Ortega}^{3}$, Bruna Grazielle Silva dos Santos ${ }^{3}$, Manoel Jacobsen Teixeira ${ }^{1,4}$

1: Division of Neurosurgery (LIM 62), Hospital das Clínicas, Faculty of Medicine, University of São Paulo, São Paulo, Brazil

2: Neurosurgery Service, Hospital Regional do Vale do Paraíba, Taubaté, Brazil

3: Department of Medicine, University of Taubaté (Unitau), Taubaté, Brazil

4: Department of Neurology, Faculty of Medicine, University of São Paulo, São Paulo, Brazil

*corresponding author: adriano.nogueira@hc.fm.usp.br, 255 Dr. Eneas de Carvalho Aguiar Ave, 05403-900, Sao Paulo, Brazil

\begin{abstract}
Evidence on adult mammalian neurogenesis and scarce studies with human brains led to the idea that adult human neurogenesis occurs in the subgranular zone (SGZ) of the dentate gyrus and in the subventricular zone (SVZ). However, findings published from 2018 rekindled controversies on adult human SGZ neurogenesis. We systematically reviewed studies published during the first decade of characterization of adult human neurogenesis (1994-2004) - when the two-neurogenic-niche concept in humans was consolidated - and compared with further studies. The synthesis of both periods is that adult human neurogenesis occurs in an intensity ranging from practically zero to a level comparable to adult mammalian neurogenesis in general, which is the prevailing conclusion. Nonetheless, Bernier and colleagues showed in 2000 intriguing indications of adult human neurogenesis in a broad area including the limbic system. Likewise, we later showed evidence that limbic and hypothalamic structures surrounding the circumventricular organs form a continuous zone expressing neurogenesis markers encompassing the SGZ and SVZ. The conclusion is that publications from 2018 on adult human neurogenesis did not bring novel findings on location of neurogenic niches. Rather, we expect that the search of neurogenesis beyond the canonical adult mammalian neurogenic niches will confirm our indications that adult human neurogenesis is orchestrated in a broad brain area. We predict that this approach may, for example, clarify that human hippocampal neurogenesis occurs mostly in the CA1subiculum zone and that the previously identified human rostral migratory stream arising from the $\mathrm{SVZ}$ is indeed the column of the fornix expressing neurogenesis markers.
\end{abstract}

Key words: neural stem cells, hypothalamus, circumventricular organs, limbic system, neuronal plasticity, hippocampus

\section{Introduction}

The discovery of adult mammalian neurogenesis was a paradigm shift in neuroscience (Altman 1962). The long-lasting idea of "no neuron formation after birth" was replaced 
by an idea of "two post-natal neurogenic niches" (Ming and Song 2011). Currently, adult mammalian neurogenesis is thought to occur primarily only in the subgranular zone (SGZ) of the dentate gyrus and in the subventricular zone (SVZ). Accordingly, neural stem cells (NSCs) in the SGZ give rise to neurons that integrate into the dentate gyrus circuitry (Ming and Song 2011) and NSCs in the SVZ give rise to neuroblasts that migrate through the rostral migratory stream (RMS) and differentiate into neurons in the olfactory bulb (Ming and Song 2011).

The discovery of adult mammalian neurogenesis triggered the search for adult human neurogenesis (Nogueira et al. 2014b). Concepts on adult human neurogenesis derive principally from rodent studies due to technical difficulties to investigate the human brain, which was the source of a relatively small number of studies (Nogueira et al. 2014b). Adult human neurogenesis was thought to be basically a more discrete adult mammalian neurogenesis (Nogueira et al. 2014b). However, recent studies rekindled the debate on the existence of adult human SGZ neurogenesis and its possible behavior in Alzheimer's disease (Boldrini et al. 2018; Sorrels et al. 2018; Moreno-Jiménez et al. 2019; Tobin et al. 2019).

To discuss the return of initial controversies on adult human neurogenesis, we established an operational classification regarding the eras of development of knowledge on adult human neurogenesis, which is the following:

1) discovery and consolidation (1994 - 2004): from the discovery of adult human neurogenesis (Kirschenbaum et al 1994) to the consolidation of the "twoneurogenic-niche" concept in humans (Ming and Song 2011);

2) corroboration of the "two-neurogenic-niche" concept (Kumar et al 2019) and controversy on the existence of the RMS (2005 - 2017) (Nogueira et al 2014b): increasing number of manuscripts that corroborated the "two-neurogenic-niche" concept, although with controversies regarding the existence of the RMS;

3) controversy on SGZ neurogenesis (2018 - present): novel studies with contradictory results on adult human neurogenesis in normal and Alzheimer's disease brains (Boldrini et al. 2018; Sorrels et al. 2018; Moreno-Jiménez et al. 2019; Tobin et al. 2019).

Here we carry out a systematic review of the first-era studies on adult human neurogenesis to show that the recent publications did not bring novelty on location of human neurogenic niches.

More important, we show that the work by Bernier et al. (2000) in the first era reporting indications of neurogenesis in the limbic system, hypothalamus, and striatum and our work in the second era (Nogueira et al. 2014b) reporting indications of neurogenesis in limbic and hypothalamic structures surrounding the circumventricular organs are alternatives to the prevailing view on adult human neurogenesis that cannot be ruled out by the third-era studies because they did not explore areas beyond the canonical mammalian neurogenic niches (Boldrini et al. 2018; Sorrels et al. 2018; MorenoJiménez et al. 2019; Tobin et al. 2019).

Indeed, we have been emphasizing that the analysis of larger brain zones may unveil a bigger picture of adult human neurogenesis (Figures 1 and 2) (Nogueira et al. 2014b; Nogueira et al. 2017c; Nogueira et al. 2018). This bigger picture may confirm our findings showing that in humans a continuous layer expressing neurogenesis markers arising from the choroid plexus reaches the SGZ via a minor branch and the Cornu 
Ammonis (CA)1-subiculum via a major branch (Nogueira et al. 2014b; Nogueira et al. 2018) and that the zone previously described as the location of the human RMS is actually the column of the fornix expressing neurogenesis markers or, alternatively, the zone encompassing the anterior hypothalamus or septal area (Nogueira et al. 2014b; Nogueira et al. 2017c; Nogueira et al. 2018).

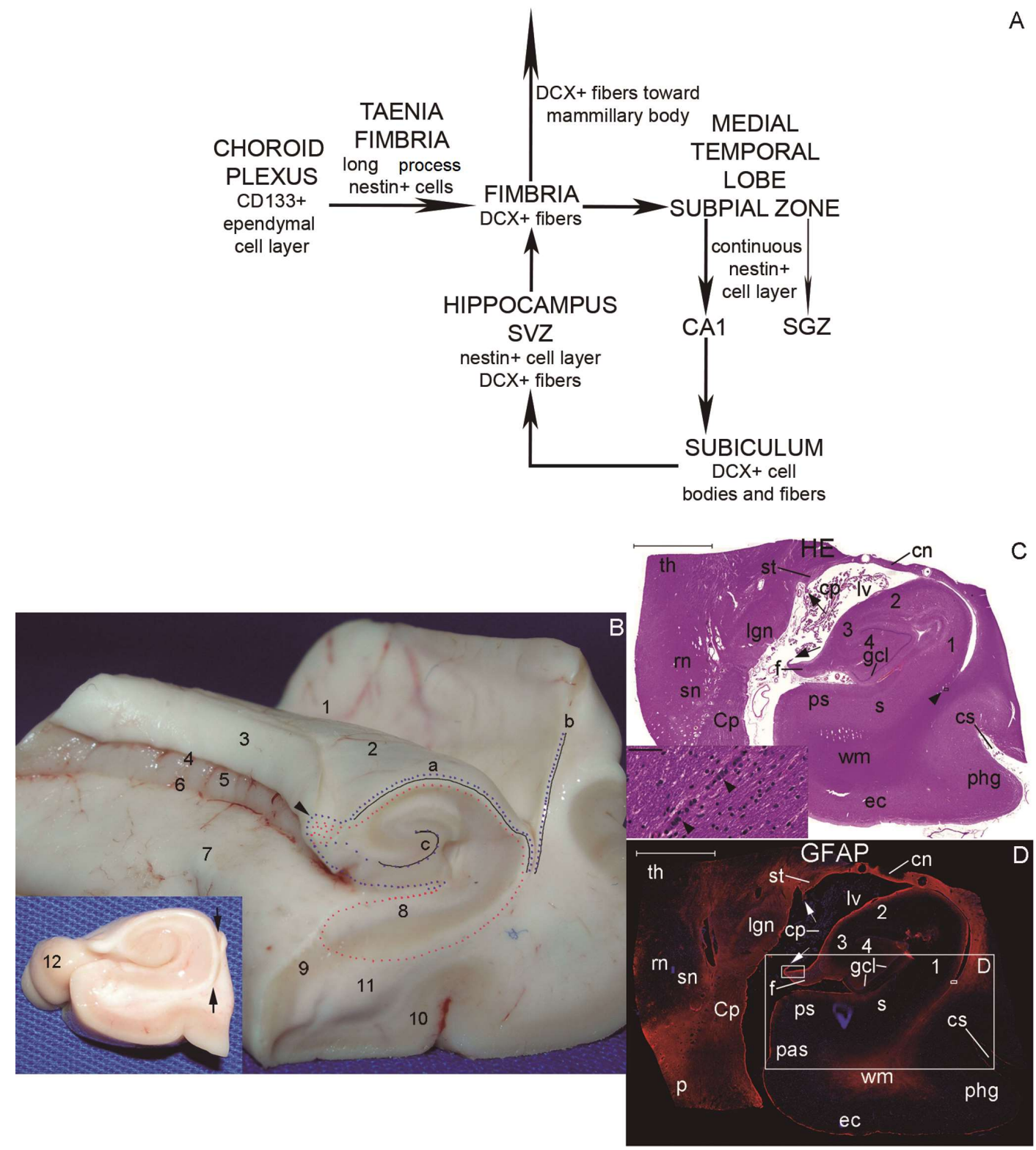

Figure 1. Structures and flux of the potential neurogenic system in the temporal lobe.

Figure $\mathrm{A}$ is a scheme of the neurogenesis flux based on our findings regarding the temporal lobe (Nogueira et al. 2014b). Figure B is an anteromedial view of the macroscopic anatomy of the medial temporal lobe sectioned at the level of the body of the hippocampus depicted with lines comparing the canonical neurogenic niches with our results (Nogueira et al. 2014b). Figures C and $\mathrm{D}$ are respectively stained by hematoxylin-eosin (HE) and the astrocyte marker glial fibrillary acidic protein (GFAP) and show a large coronal view of the structures of the cytoarchitecture of the medial temporal lobe captured by a slide scanner. The choroid plexus (cp) is regarded a circumventricular organ. Choroid plexus ependymal cells express the neural 
stem cell marker CD133. The choroid plexus is attached to the fimbria (f) by the taenia fimbria (arrowhead in B and arrows in C and D), which harbors nestin+ cells with long processes. From the fimbria, a continuous nestin+ cell layer (represented by blue dotted lines in B) follows through the SVZ of the temporal horn of the lateral ventricle and through the subpial layer of the medial temporal lobe. This layer forks as a minor branch toward the SGZ and as a major branch ending at the CA1 zone. Adjacent to this zone (dotted red lines in B) - at the subiculum $-\mathrm{DCX}+$ cell bodies send DCX + cell fibers that contour the subicular complex, follow across the SVZ covering the hippocampus and next across the fimbria up to the border of the brain slices analyzed by Nogueira et al. (2014b). Black lines identify the canonical neurogenic niches, namely, a, hippocampal SVZ; b, SVZ (collateral eminence); c, SGZ. $1-4$ in C and D refers to CA1 - CA4. Arrowheads in C and its inset point to an intraparenchymal ependymal cell layer (Nogueira et al. 2014b). Rectangle D with two smaller rectangles refer to zone with images amplified in our original paper (Nogueira et al. 2014b). Legend: in B, 1, collateral eminence; 2 , body of the hippocampus; 3 (and arrowhead), fimbria; 4, fimbriodentate sulcus; 5, dentate gyrus (margo denticulatus); 6, hippocampal sulcus; 7, parahippocampal gyrus (medial surface) (macroscopic anatomy); 8, subiculum; 9, entorhinal cortex (presubiculum and parasubiculum are located between 8 and 9); 10, parahippocampus (cytoarchitecture); 11, parahippocampal gyrus (coronal cut) (macroscopic anatomy); 12, uncus. In C and D, cn, tail of the caudate nucleus; $\mathrm{Cp}$, cerebral peduncle; cs, collateral sulcus; ec, entorhinal cortex; gcl, granule cell layer of the dentate gyrus; lgn, lateral geniculate nucleus; lv, lateral ventricle; p, pons; pas, parasubiculum; phg, parahippocampal gyrus (cytoarchitecture definition); ps, presubiculum; rn, red nucleus; s, subiculum; sas, subarachnoid space; sn, substantia nigra; st, stria terminalis; th, thalamus; wm, white matter of the parahippocampal gyrus (gross anatomy definition). Scale bars: 5,000 $\mu \mathrm{m}$. Figure adapted from Nogueira et al. (2014b) under CC BY 2.0 license.

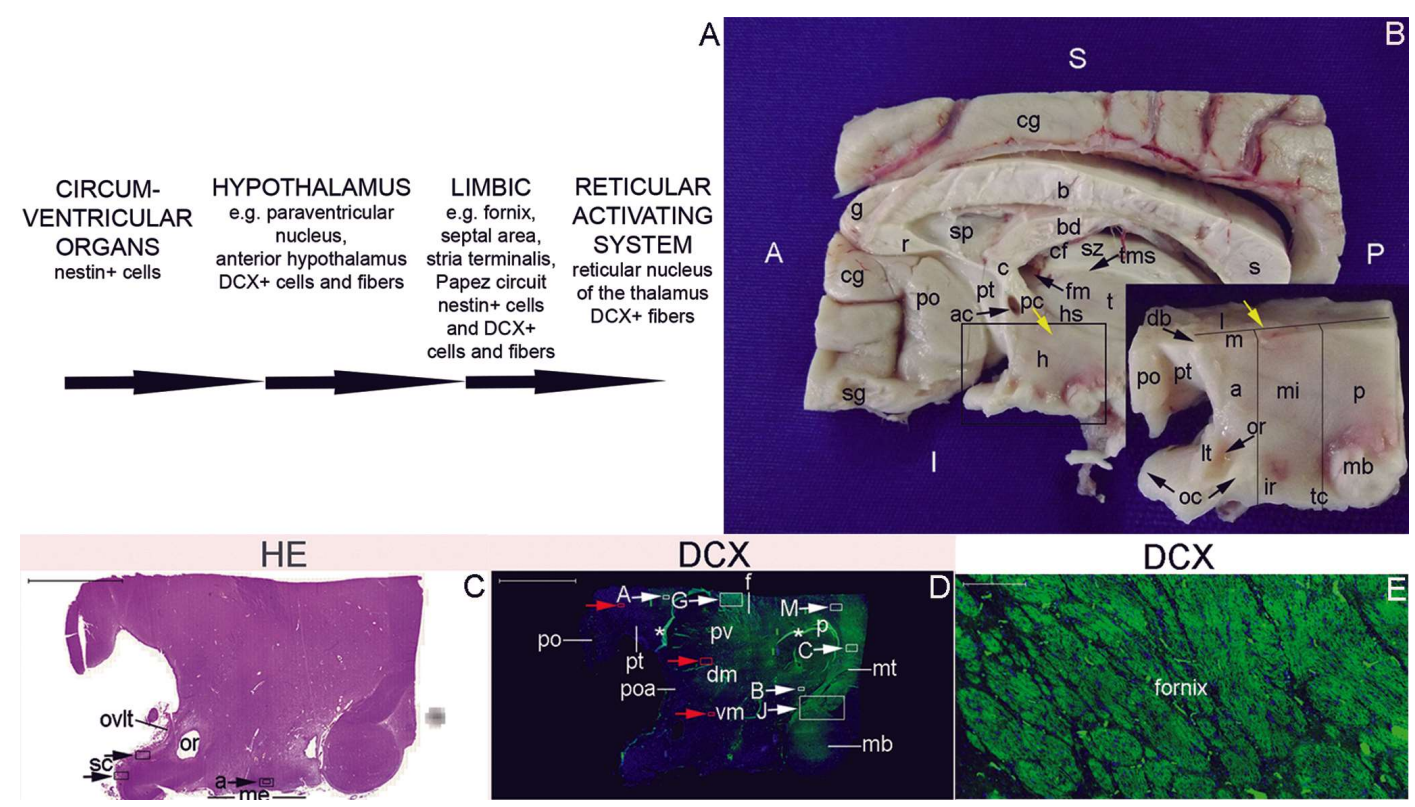

Figure 2. Structures and flux of the potential neurogenic system. Figure $A$ is a scheme of our findings (Nogueira et al. 2014b) showing that broadly the expression of markers of degree of differentiation of neuronal cells parallels an anatomical and functional flux. We found indications of this flux related to $1^{\text {st }}$ ) homeostasis in the circumventricular organs such as the median eminence (me), the organum vasculosum laminae terminalis (ovlt), and the subfornical organ; $2^{\text {nd }}$ ) autonomic regulation at, for example, the anterior hypothalamus and paraventricular nucleus ( $\mathrm{pv}) ; 3^{\text {rd }}$ ) emotion related to limbic structures such as the fornix, mammillary body $(\mathrm{mb})$, and septal area (paraterminal gyrus ( $\mathrm{pt})$ plus paraolfactory gyrus (po)); and $4^{\text {th }}$ ) arousal influenced by the nucleus reticularis of the thalamus. Figure B is a macroscopic view of the core 
of the potential neurogenic system, and the inset shows the sample dissected by cuts delimited by the rectangle in the large figure and studied in Figures $\mathrm{C}-\mathrm{E}$. Vertical lines in the inset delimitates the anterior (a), middle (mi), and posterior (p) hypothalamus parts. Rectangles labeled in C and D correspond to zones that we presented somewhere else (Nogueira et al. 2014 b) with larger magnification images. Note that the column of the fornix (c and yellow arrows in B, f in D, and Figure E) displays a dense expression of DCX that could have been misinterpreted as the location of the RMS principally if the surrounding structures are not analyzed on the same slice. Legend: A, anterior; ac, location of the anterior commissure; b, body of the corpus callosum; bd, body of the fornix; cf, choroidal fissure; cg, cingulate gyrus; $\mathrm{db}$, diagonal band; $\mathrm{dm}$, dorsomedial nucleus zone; fm, forame of Monro; $\mathrm{g}$, genu of the corpus callosum; h, hypothalamus; HE, hematoxylin and eosin; hs, hypothalamic sulcus; I, inferior; ir, infundibular recess; 1, lateral hypothalamic zone; lt, lamina terminalis; m, medial hypothalamic zone; mt, mammillothalamic tract; oc, optic chiasm; or, optic recess; P, posterior; pc, postcommissural fornix; po, pre-optic area; pv, paraventricular nucleus zone; r, rostrum of the corpus callosum; S, superior; s, splenium of the corpus callosum; sc, supra-chiasmatic nucleus zone; sg, straight gyrus; sp, septum pellucidus; sz, stratum zonale; t, thalamus; tc, tuber cinereo; tms, thalamic medial striae; vm, ventromedial nucleus zone. Asterisks identify artifacts. Scale bars: B and C: 5,000 $\mu \mathrm{m}$; D: $200 \mu \mathrm{m}$. Figure adapted from Nogueira et al. (2014b) under CC BY 2.0 license.

\section{Systematic review of early studies on adult human neurogenesis (1994- 2004)}

\subsection{Searching method and number of original articles}

The initial source used to gather the references was Medline (Pubmed), comprising papers published up to 2004. Each one of the key-words "neural stem cells", "neural progenitor cells", and "neurogenesis" were intersected with "adult and mammals" or "human"; the words "progenitor cells" and "stem cells" were separately intersected with "Alzheimer", "demyelinating”, "head injury", "Huntington", "neurological diseases", "Parkinson", "spinal injury", and "stroke". We found 21 original articles on adult human neurogenesis (Kirschenbaum et al. 1994; Pincus et al. 1997; Eriksson et al. 1998; Pincus et al. 1998; Johansson et al. 1999; Kukekov et al. 1999; Bernier et al. 2000; Pagano et al. 2000; Roy et al. 2000a; Roy et al. 2000b; Akiyama et al. 2001; Arsenijevic et al. 2001; Blümcke et al. 2001; Palmer et al. 2001; Curtis et al. 2003; Liu and Martin 2003; Nunes et al. 2003; Westerlund et al. 2003; Bédard and Parent 2004; Jin et al. 2004; Sanai et al. 2004). The first article was published in 1994 (Kirschenbaum et al 1994).

\subsection{Sources of adult human NSCs}

Fifteen studies used brain tissue resected from neurosurgical patients (Table 1)

(Kirschenbaum et al. 1994; Pincus et al. 1997; Pincus et al. 1998; Johansson et al. 1999; Kukekov et al. 1999; Pagano et al. 2000; Roy et al. 2000a; Roy et al. 2000b; Akiyama et al. 2001; Arsenijevic et al. 2001; Blümcke et al. 2001; Palmer et al. 2001; Nunes et al. 2003; Westerlund et al. 2003; Sanai et al. 2004). In most cases, the resections came from epileptic patients (13 articles) (Table 1) (Kirschenbaum et al. 1994; Pincus et al. 1997; Pincus et al. 1998; Johansson et al. 1999; Kukekov et al. 1999; Roy et al. 2000a; Roy et al. 2000b; Arsenijevic et al. 2001; Blümcke et al. 2001; Palmer et al. 2001; Nunes et al. 2003; Westerlund et al. 2003; Sanai et al. 2004), all of which with samples of temporal lobe epilepsy non-secondary to tumor or dysplasia. The number of patients with temporal lobe epilepsy was 105. Eleven groups (Eriksson et al. 1998; Kukekov et al. 1999; Bernier et al. 2000; Akiyama et al. 2001; Blümcke et al. 2001; Palmer et al. 
2001; Curtis et al. 2003; Liu and Martin 2003; Bédard and Parent 2004; Jin et al. 2004; Sanai et al. 2004) used a total of 143 specimens from brains obtained in autopsy.

Table 1. Methods: detection of adult human neural stem cells (source of tissues and locations)

Reference

Source of Tissues

\section{Location}

Temporal lobe (Cx, WM, SVZ)

(1994)

Pincus et al. $\quad 8$ epileptic patients

(1997)

Pincus et al. $\quad 16$ epileptic patients (in vivo

(1998) studies -5 specimens)

Eriksson et al. (1998)

5 cancer patients (nonneurological)

Kukekov et al. 10 autopsies; (1999) 10 epileptic patients (temporal lobe)

Johansson et al. 2 epileptic patients

(1999)

Bernier et al. $\quad 19$ autopsies (2000)

Roy et al.

(2000b)

Roy et al.

(2000a)

Pagano et al.

(2000)

Akiyama et al.

(2001)

Arsenijevic et al. (2001)

Blümcke et al. (2001)
8 patients: epilepsy, 4; meningioma, 2; aneurysm, 1 ; traumatic edema, 1 4 patients: epilepsy, 3 ; head injury, 1

Olfactory bulb (neurosurgical patients)

5 tumor patients (glioma); 1 autopsy

13 patients: epilepsy (temporal, 7; frontal, 1; frontal displasia, 2); head injury (2); brain edema (1)

22 epileptic patients (comprising the temporal lobe); 4 CNS tumor
Temporal lobe (CX, SVZ)

Temporal lobe (in vitro: CX, SVZ; In vivo: SVZ)

Temporal lobe (Hc)

SVZ (peristriatal / perihippocampal: autopsies);

Temporal lobe (in vitro: $\mathrm{Cx}, \mathrm{SVZ}, \mathrm{Hc}$ )

Temporal lobe (SVZ, Hc)

SVZ

Temporal lobe (dentate gyrus)

Temporal lobe (SVZ)

Olfactory bulb

Tumor patients: temporal lobe $(C x, 1$; $\mathrm{HC}, 1)$; frontal lobe (Cx, 1; SVZ, 2);

Autopsy: frontal lobe (CX, SVZ); Hc

Temporal lobe ( $\mathrm{Cx}, 8 ; \mathrm{Hc}, 1 ; \mathrm{Amy}, 1 ; \mathrm{Hc}+$ Amy, 2; SEZ, 3); frontal lobe $(C x, 3)$

Temporal lobe (Hc) 


\begin{tabular}{|c|c|c|}
\hline & $\begin{array}{l}\text { patients (no lesion in the } \mathrm{Hc} \text { ); } 6 \\
\text { adult autopsies }\end{array}$ & \\
\hline $\begin{array}{l}\text { Palmer et al. } \\
(2001)\end{array}$ & $\begin{array}{l}1 \text { autopsy (11-week old child; } \\
\text { myofibromatosis); } 1 \text { epileptic } \\
\text { patient }\end{array}$ & $\begin{array}{l}\text { Post-mortem tissue (in vitro study): Hc, } \\
\text { SEZ, motor Cx, corpus callosum; } \\
\text { epileptic: temporal lobe (Cx, WM, Hc) }\end{array}$ \\
\hline $\begin{array}{l}\text { Curtis et al. } \\
(2003)\end{array}$ & $\begin{array}{l}13 \text { autopsies ( } 9 \text { Huntigton's } \\
\text { disease patients; } 4 \text { controls) }\end{array}$ & SEZ adjacent to caudate nucleus \\
\hline $\begin{array}{l}\text { Liu and Martin } \\
\text { (2003) }\end{array}$ & 10 autopsies & Olfactory bulb \\
\hline $\begin{array}{l}\text { Nunes et al. } \\
(2003)\end{array}$ & $\begin{array}{l}21 \text { patients: epilepsy (14), } \\
\text { aneurysm (1), displasia (2), post- } \\
\text { traumatic decompression (4) }\end{array}$ & Temporal lobe (WM) \\
\hline $\begin{array}{l}\text { Westerlund et al. } \\
(2003)\end{array}$ & 4 epileptic patients & Temporal lobe (SVZ) \\
\hline $\begin{array}{l}\text { Bédard and Parent } \\
\text { (2004) }\end{array}$ & 10 autopsies & Olfactory bulb \\
\hline Jim et al. (2004) & $\begin{array}{l}25 \text { autopsies ( } 14 \text { Alzheimer's } \\
\text { disease patients; } 9 \text { controls) }\end{array}$ & $\mathrm{Hc}$ \\
\hline Sanai et al. (2004) & $\begin{array}{l}65 \text { neurosurgical patients; } 45 \\
\text { autopsies* }\end{array}$ & $\begin{array}{l}\text { SEZ (temporal and extra-temporal, 110); } \\
\text { temporal (10) and parietal ( } 8 \text { ) Cx; } \\
\text { septum (in vitro: } 3 \text {; in vivo: } 6 \text { ); striatum } \\
\text { (14); olfactory tract (11) }\end{array}$ \\
\hline
\end{tabular}

* Excluding olfactory tract specimens. Amy, amygdala; BrdU, bromodeoxyuridine; CNS, central nervous system; Cx, cortex; Hc, hippocampus; SVZ, subventricular zone; WM, white matter.

\subsection{Methods used in the original articles on adult human neurogenesis}

The studies carried out in vivo and retrospective and prospective in vitro methods. In vivo methods corresponded principally to immunostaining for neurogenesis markers performed on brain slices (Tables 2 and 3). Retrospective in vitro methods were used in 11 papers (Tables 4 and 5) (Kirschenbaum et al. 1994; Pincus et al. 1997; Pincus et al. 1998; Johansson et al. 1999; Kukekov et al. 1999; Pagano et al. 2000; Akiyama et al. 2001; Arsenijevic et al. 2001; Palmer et al. 2001; Westerlund et al. 2003; Sanai et al. 2004 ) and consisted basically in neurosphere assay. Prospective in vitro study was applied in three articles (Table 6) (Roy et al. 2000a; Roy et al. 2000b; Nunes et al., 2003). In these articles, cell sorting was carried out by transfection of plasmid DNA encoding the gene for human green fluorescent protein placed under the control of regulatory sequences of genes encoding the immature neuron marker T $\alpha 1$-tubulin, the NSC marker nestin (Roy et al. 2000a; Roy et al. 2000b), and CNP2 (Nunes et al. 2003), which selects white matter progenitor cells, also selected by fluorescent or immunomagnetic methods of separation using A2B5 as marker. 
Table 2. In vivo methods used to detect adult human neural stem cells

Reference Markers

Pincus et al. Committed (Hu protein) and uncommitted (musashi protein) progenitor cells (1998)

Eriksson et al. Cell Mt/Pr (BrdU);

(1998) Nr (NeuN, NSE, calbindin); At (GFAP)

Kukekov et al. Cell Mt/Pr (Ki-67); extracellular matrix protein (tenascin-C);

(1999) At (GFAP)

Bernier et al. Cell Mt/Pr (PCNA); anti-apoptotic protein (Bcl-2); NSC (nestin);

(2000) Nr ( $3 I I I$ t, PSA-NCAM); At (GFAP)

Akiyama et al. NSC (nestin)

(2001)

Blümcke et al. NSC (nestin); GPC (vimentin); Nr ( $\beta$ IIIt, MAP-2, MAP 1b/5, NeuN, NF-L,

(2001) calbindin); At (GFAP, S100ß); M $\varnothing$ (CD 68 / HLA-DR); leukocytes (CD45)

Curtis et al. $\quad$ Cell Mt/Pr (PCNA); Nr ( $(\beta I I t$, NeuN); At (vimentin, GFAP)

(2003) (Western Blott: PCNA; TUNEL)

Liu and Martin Early post-mitotic neuron (TUC-4); NSC (nestin); GPC (vimentin);

(2003) $\mathrm{Nr}(\beta I I I t, \mathrm{NeuN})$; At (GFAP)

Bédard and Parent (2004)

Cell Mt/Pr (Ki-67, PCNA), NSC (nestin); anti-apoptotic protein (Bcl-2); migrating neuroblasts (DCX); periglomerular interneurons (CR, GAD $65 / 67, \mathrm{PV})$; granular interneurons $\left(\mathrm{GAD}_{65 / 67}\right)$; dopamine cell marker $(\mathrm{TH})$; $\mathrm{Nr}$ (NeuroD, PSA-NCAM, $\beta I I I t)$

Jim et al. (2004) Migrating neuroblasts (DCX), committed progenitor cells (Hu protein), early post-mitotic neuron (TUC-4), Nr (PSA-NCAM, , NeuN, NeuroD, calbindin), AD $\left(A \beta_{1-17}\right.$, epitopes of human APP (5A3 and $\left.1 G 7\right)$, phospho- $\tau$ ), programmed cell death (cleaved caspase-8);

(Western Blott: antibodies: DCX, PSA-NCAM, NeuN, TUC-4, NeuroD, calbindin, actin)

Sanai et al. $\quad$ Cell Mt/Pr (BrdU, Ki-67); GPC (vimentin); Nr (PSA-NCAM, $\beta$ IIIt); At (GFAP); (2004) Oligodendrocytes (O4)

$A D$, histopathological hallmarks of Alzheimer's disease; APP, amyloid precursor protein; At, astrocyte; $A \beta, \beta$ amyloid peptide; BrdU, Bromodeoxyuridine; Cell Mt/Pr, cellular mitosis / proliferation; CR, calretinin; DCX, doublecortin; GAD, glutamic acid decarboxylase; GFAP, glial fibrillary acid protein; GPC, glial progenitor cell; Hc, hippocampus, MAP, microtubuleassociated protein; $M \varnothing$, macrophage/microglia; NeuN, neuron-specific nuclear protein; NF-L, neurofilament; Nr, neuron; NSC, neural stem cell; NSE, neuron specific enolase; PCNA, proliferating cell nuclear antigen; PSA-NCAM, polysialylated form of neural cell adhesion 
molecule; PV, parvalbumin; TH, tyrosine hidroxylase; TUC-4, turned on / Unc-33-like phosphoprotein-1 / CRMP-4 family; TUNEL, terminal deoxynucleotidyltransferase-mediated dATP nick and labeling; $\beta$ IIIt, $\beta$ III-tubulin.

Table 3. In vivo methods (phenotypes $\mathrm{X}$ markers $\mathrm{X}$ locations)

\begin{tabular}{|c|c|c|}
\hline Phenotypes & Markers & Locations \\
\hline \multirow[t]{7}{*}{ Immature / progenitor cells } & $\mathrm{Hu}$ & $\mathrm{HC}(\mathrm{a}), \mathrm{SVZ}(\mathrm{b})$ \\
\hline & Musashi & SVZ (b) \\
\hline & Nestin & $\mathrm{Hc}(\mathrm{a})(\mathrm{b}), \mathrm{OB}(\mathrm{a}), \mathrm{FL}(\mathrm{Cx}, \mathrm{SVZ})$ \\
\hline & & (b), SVZ (a) \\
\hline & Vimentin & Hc (a) (b), OB (a), SVZ (a) (b) \\
\hline & Tenascin-C* & SVZ (a) \\
\hline & $\mathrm{Bcl}-2$ & OB (a), SVZ (a) \\
\hline \multirow[t]{4}{*}{ Cellular mitosis / proliferation } & BrdU & Hc (a), SVZ (a) (b) \\
\hline & Ki-67 & OB (a), SVZ (a) (b) \\
\hline & TUC-4 & $\mathrm{OB}(\mathrm{a})$ \\
\hline & PCNA & OB (a), Hc (a), SVZ (a) \\
\hline \multirow[t]{15}{*}{ Neuronal lineage } & $\beta$ III-tubulin & Hc (a) (b), SVZ (a) (b), OB (a) \\
\hline & AD halmarks & $\mathrm{Hc}(\mathrm{a})$ \\
\hline & $\mathrm{CR}$ & $\mathrm{OB}(\mathrm{a})$ \\
\hline & PV & $\mathrm{OB}(\mathrm{a})$ \\
\hline & DCX & $\mathrm{OB}(\mathrm{a})$ \\
\hline & GAD & $\mathrm{OB}(\mathrm{a})$ \\
\hline & MAP-2 & $\mathrm{Hc}(\mathrm{a})(\mathrm{b})$ \\
\hline & MAP $1 b / 5$ & $\mathrm{Hc}(\mathrm{a})(\mathrm{b})$ \\
\hline & NeuN & Hc (a) (b), OB (a), SVZ (a) \\
\hline & NeuroD & $\mathrm{Hc}(\mathrm{a}), \mathrm{OB}(\mathrm{a})$ \\
\hline & NF-L & $\mathrm{Hc}(\mathrm{a})(\mathrm{b})$ \\
\hline & Calbindin & $\mathrm{Hc}(\mathrm{a})(\mathrm{b})$ \\
\hline & PSA-NCAM & Hc (a), OB (a), SVZ (a) (b) \\
\hline & NSE & $\mathrm{Hc}(\mathrm{a})$ \\
\hline & $\mathrm{TH}$ & $\mathrm{OB}(\mathrm{a})$ \\
\hline \multirow[t]{2}{*}{ Astrocytes } & GFAP & Hc (a) (b), SVZ (a) (b), OB (a) \\
\hline & S100 $\beta$ & $\mathrm{Hc}(\mathrm{a})(\mathrm{b})$ \\
\hline \multirow[t]{5}{*}{ Others } & CD68 (M $\varnothing /$ microglia) & $\mathrm{Hc}(\mathrm{a})(\mathrm{b})$ \\
\hline & HLA-DR (M $\varnothing /$ microglia) & $\mathrm{Hc}(\mathrm{a})(\mathrm{b})$ \\
\hline & CD-45 (leukocytes) & $\mathrm{Hc}(\mathrm{a})(\mathrm{b})$ \\
\hline & $\begin{array}{l}\text { Cleaved caspase- } 8 \\
\text { (programmed cell death) }\end{array}$ & $\mathrm{Hc}(\mathrm{a})$ \\
\hline & O4 (oligodendrocytes) & SVZ (a) (b) \\
\hline \multicolumn{3}{|c|}{$\begin{array}{l}\text { *development-related extracellular matrix protein. a, autopsy; } A D \text {, Alzheimer's disease }\left(A \beta_{1-17} \text {, }\right. \\
5 A 3,1 G 7 \text {, phospho- } \tau \text { ); b, biopsy; Bcl-2, product of the antiapoptotic gene } B c l-2 ; B r d U, \\
\text { bromodeoxyuridine; CR, calretinin; Cx, cortex; } D C X \text {, doublecortin; FL, frontal lobe; GAD, } \\
\text { glutamic acid decarboxylase; GFAP, glial fibrillary acid protein; Hc, hippocampus; MAP, }\end{array}$} \\
\hline
\end{tabular}


microtubule-associated protein; $M \varnothing$, macrophage; NeuN, neuron-specific nuclear protein; NF$L$, neurofilament; NSE, neuron specific enolase; OB, olfactory bulb; PCNA, proliferating cell nuclear antigen; PSA-NCAM, polysialylated form of neural cell adhesion molecule; PV, parvalbumin; SVZ, subventricular zone; TH, tyrosine hydroxylase ; TUC-4, turned on / Unc-33like phosphoprotein-1/ CRMP-4 family.

Table 4. Retrospective in vitro methods used to detect adult human neural stem cells

\begin{tabular}{|c|c|c|c|}
\hline Reference & Culture Media & Immunostaining & Others \\
\hline $\begin{array}{l}\text { Kirschenbaum et al. } \\
\text { (1994) }\end{array}$ & DMEM/F12; EGF & $\begin{array}{l}\mathrm{Nr} \text { (MAP-2, MAP-5, NF, } \\
\mathrm{N}-\mathrm{CAM}) ; \text { At (GFAP); O } \\
(\mathrm{O} 4, \mathrm{O} 1), \mathrm{pro}-\mathrm{O}(\mathrm{A} 2 \mathrm{~B} 5 \text {, } \\
\left.\mathrm{G}_{\mathrm{D} 3}, \mathrm{O} 4\right) ; \mathrm{MGl} \text { (CD68) }\end{array}$ & $\begin{array}{l}\text { Calcium imaging } \\
\text { Autoradiography }\end{array}$ \\
\hline $\begin{array}{l}\text { Pincus et al. } \\
\text { (1997) }\end{array}$ & $\begin{array}{l}\text { DMEM/F12/N2; } \\
\text { FGF-2, BDNF }\end{array}$ & $\mathrm{Nr}(\mathrm{MAP}-2)$ & $\begin{array}{l}\text { Calcium imaging } \\
\text { Autoradiography }\end{array}$ \\
\hline $\begin{array}{l}\text { Pincus et al. } \\
\text { (1998) }\end{array}$ & $\begin{array}{l}\text { DMEM/F12/N2; } \\
\text { FGF-2, BDNF (+Ctrl) }\end{array}$ & $\mathrm{Nr}(\mathrm{MAP}-2)$ & $\begin{array}{l}\text { Calcium imaging } \\
\text { Autoradiography }\end{array}$ \\
\hline $\begin{array}{l}\text { Kukekov et al. } \\
\text { (1999) }\end{array}$ & $\begin{array}{l}\text { DMEM/F12; } \\
\text { No growth factors }\end{array}$ & $\begin{array}{l}\text { NSC / At (nestin); INr/At } \\
\text { (vimentin); Mt (BrdU); } \\
\text { Pax-6*; Bcl-2; tenascin- } \\
\text { C; At (GFAP); Nr ( } \beta \text { IIIt, } \\
\text { L1) }\end{array}$ & $\begin{array}{l}\text { Electron microscopy; } \\
\text { PCR (nestin, NF-M, NSE, } \\
\text { GFAP, tenascin, Pax-6, } \\
p l p, d m-20 \text { ) }\end{array}$ \\
\hline $\begin{array}{l}\text { Johansson et al. } \\
\text { (1999) }\end{array}$ & $\begin{array}{l}\text { DMEM / F12; EGF, } \\
\text { FGF-2; NS d/r }\end{array}$ & $\begin{array}{l}\text { At (GFAP), } \mathrm{Nr}(\beta \| \mathrm{It}) \text {, } \\
\mathrm{O}(\mathrm{O} 4)\end{array}$ & \\
\hline $\begin{array}{l}\text { Pagano et al. } \\
(2000)\end{array}$ & $\begin{array}{l}\text { DMEM / F12; EGF, } \\
\text { FGF-2, LIF-1; } \\
\text { NS d/r }\end{array}$ & $\begin{array}{l}\text { At (GFAP), Nr ( } \beta \| l \mathrm{t}) \text {, } \\
\text { O (galactocerebroside) }\end{array}$ & \\
\hline $\begin{array}{l}\text { Akiyama et al. } \\
\text { (2001) }\end{array}$ & $\begin{array}{l}\text { NPMM; EGF, FGF-2, } \\
\text { NS d/r (+2 passages) }\end{array}$ & $\begin{array}{l}\text { GPC (vimentin), IG } \\
\text { (A2B5), NSC (nestin), At } \\
\text { (GFAP, S-100), Nr ( } \beta \text { IIIt, } \\
\text { MAP-2, NF, NSE), O (O4, } \\
\text { galactocerebroside), } \\
\text { Sch(P0) }\end{array}$ & $\begin{array}{l}\text { Chimeras: AHNSC } \\
\text { transplant in rodent } \\
\text { demyelinated spine } \\
\text { (neurophysiological and } \\
\text { immunostaining) }\end{array}$ \\
\hline $\begin{array}{l}\text { Arsenijevic et al. } \\
\text { (2001) }\end{array}$ & $\begin{array}{l}\text { DMEM / F12; EGF, } \\
\text { FGF-2, BDNF, PDGF; } \\
\text { NS d/r }\end{array}$ & $\begin{array}{l}\text { NSC (nestin), Mt (BrdU), } \\
\text { At (GFAP), Nr ( } \beta \text { IIIt, } \\
\text { MAP-2, N-CAM, Neu-N), } \\
\text { O (O4); clonal } \\
\text { origin (lac-Z) }\end{array}$ & $\begin{array}{l}\text { PCR: } \\
\text { (detection of lentiviral } \\
\text { inserction ) }\end{array}$ \\
\hline $\begin{array}{l}\text { Palmer et al. } \\
\text { (2001) }\end{array}$ & $\begin{array}{l}\text { DMEM / F12; } \\
\text { EGF, FGF-2, PDGF; } \\
25 \% \text { modified stem cells } \\
\text { conditioned medium }\end{array}$ & $\begin{array}{l}\text { At (GFAP), IG (A2B5), } \\
\mathrm{Nr}(\beta \mathrm{IIIt}, \mathrm{NF150,} \text { MAP-2), } \\
\mathrm{O}(\mathrm{O} 4)\end{array}$ & \\
\hline
\end{tabular}


Westerlund et al. (2003)

Sanai et al. (2004)
DMEN/F12;
EGF, FGF-2

DMEN/F12; EGF; FGF-2; 50,000 cells $/ \mathrm{ml}$ in culture media
NSC (nestin); Nr ( $\beta$ IIIt, Neurophysiology: patchNeuN); At (GFAP); O clamp

(04)

$\mathrm{Mt}(\mathrm{BrdU}) ; \mathrm{Nr}(\beta \mathrm{III}) ;$ At Electron microscopy; (GFAP); O (O4) Microdissected SVZ in medium with BrdU; Prospective component of the paper: cells incubated with GFAPpGFP adenovirus, to separete SVZ At

* developmentally regulated transcription factor; $\mathrm{Bcl}-2$, product of the antiapoptotic gene $\mathrm{BCl}$ 2; BDNF, brain derived neurotrophic factor; BrdU, Bromodeoxyuridine; Ctrl, control group; DMEM, Dulbecco's modified Eagle's medium; EGF, epithelial growth factor; FGF-2, basic fibroblast growth factor ; GFAP, glial fibrillary acid protein; GFAPp, GFAP promoter; GFP, green fluorescent protein; GPC, glial progenitor cells; IG, immature glia; INr/At, immature neurons and astrocytes; lac-Z, $\beta$-galactosidase; LIF-1, leukemia inhibitory factor; L1, adhesion molecule L1; MAP, microtubule-associated protein; MGI, microglia; Mt, mitosis; N-CAM, neural cell adhesion molecule; Neu-N, neuron-specific nuclear protein NF, neurofilament; NPMM, neural progenitor cell maintenance medium; $\mathrm{Nr}$, neuron; $\mathrm{NS} d / r$, neurosphere division and replating; NSC, neural stem cells; NSE, neuron specific enolase; O, oligodendrocyte; PDGF, platelet derived growth factor; PCR, polymerase chain reaction; Sch, Schwann cell; SVZ, subventricular zone; $\beta$ IIIt, $\beta$ III-tubulin.

Table 5. Retrospective in vitro methods (phenotype $\mathrm{X}$ markers)

\section{Phenotypes}

Immature / progenitor cells

Cellular mitosis / proliferation

Clonal origin

Neuronal lineage
Markers

Nestin

Vimentin

A2B5

Pax-6*

Bcl-2

Tenascin-C

BrdU

LacZ

$\beta$ III-tubulin

MAP-2

MAP-5

NF

N-CAM

NSE

Adhesion molecule L1

NeuN

GFAP 
S100 protein

Oligodendroglial lineage

04

01

Galactocerebroside

$\mathrm{G}_{\mathrm{D} 3}$

Schwann cells

PO

Microglia

CD68

* developmentally regulated transcription factor; $\mathrm{Bcl}-2$, product of the antiapoptotic gene $\mathrm{Bcl}-$ 2; BrdU, bromodeoxyuridine; GFAP, glial fibrillary acid protein; lac-Z, $\beta$-galactosidase; MAP, microtubule-associated protein; N-CAM, neural cell adhesion molecule; Neu-N, neuron-specific nuclear protein; NF, neurofilament; NSE, neuron specific enolase

Table 6. Prospective methods used to detect adult human neural stem cells

\begin{tabular}{|c|c|c|c|}
\hline Reference & Prospective Method & Culture Media & $\begin{array}{l}\text { Immunostaining / } \\
\text { Others }\end{array}$ \\
\hline $\begin{array}{l}\text { Roy et al. } \\
(2000 b)\end{array}$ & $\begin{array}{l}\text { FACS: } \\
\text { P/T } \alpha 1: \text { hGFP or } \\
\text { E/nestin:EGFP }\end{array}$ & $\begin{array}{l}\text { DMEM/F12/N2 } \\
\text { FGF-2, BDNF }\end{array}$ & $\begin{array}{l}\text { BIII-tubulin, MAP-2, } \\
\text { GFAP, O4, BrdU; } \\
\text { Calcium imaging; } \\
\text { Patch clamp }\end{array}$ \\
\hline $\begin{array}{l}\text { Roy et al. } \\
(2000 a)\end{array}$ & $\begin{array}{l}\text { FACS: } \\
\text { P/T } \alpha 1: \text { hGFP or } \\
\text { E/nestin:EGFP }\end{array}$ & $\begin{array}{l}\text { DMEM/F12/N2 } \\
\text { FGF-2 }\end{array}$ & $\begin{array}{l}\text { BrdU, nestin, } \\
\text { MAP-2, } \beta \text { III-tubulin }\end{array}$ \\
\hline $\begin{array}{l}\text { Nunes et al. } \\
(2003)\end{array}$ & $\begin{array}{l}\text { FACS: } \\
\text { pCNP2:hGFP; } \\
\text { A2B5. } \\
\text { IMS (A2B5+ cells); } \\
\text { (obs: clonal origin: } \\
\text { A2B5+ cells tagged with } \\
\text { a lentivirus expressing } \\
\text { GFP under CMV } \\
\text { promoter) }\end{array}$ & $\begin{array}{l}\text { DMEN/F12/N1 } \\
\text { FGF-2, NT3, PDGF; } \\
\text { Plating at 50,000 cells / } \\
0.5 \mathrm{ml} \text {; primary NS } \\
\text { dissociated and } \\
\text { replated; } \\
\text { Culture in wells with } \\
\text { 1,000-100,000 cells/ml; } \\
\text { Culture of A2B5- cells }\end{array}$ & $\begin{array}{l}\text { A2B5, O4, GFAP, } \beta \text { III- } \\
\text { tubulin, MAP-2, Hu } \\
\text { protein, BrdU; } \\
\text { GAD67; HNA, nestin, } \\
\text { doublecortin; } \\
\text { Calcium imaging; } \\
\text { Patch clamp; } \\
\text { TRAP; } \\
\text { Chimera (human WMPC } \\
\text { in fetal rat brain) }\end{array}$ \\
\hline
\end{tabular}

BDNF, brain derived neurotrophic factor; BrdU, bromodeoxyuridine; DMEM, Dulbecco's modified Eagle's medium; (E)GFP, enhanced GFP; E/nestin, nestin enhancer; FACS, fluorescentactivated cell sorting; FGF-2, basic fibroblast growth factor; GAD67, glutamic acid decarboxylase-67; GFAP, glial fibrillary acid protein; hGFP, humanized green fluorescent protein; HNA, human nuclear antigen; IMS, immunomagnetic sorting; MAP, microtubuleassociated protein; NS, neurospheres; pCNP2, promoter for CNP (early oligodendrocytic transcript); PDGF, platelet-derived growth factor; P/T $\alpha 1$, T $\alpha 1$ tubulin promoter; TRAP, telomerase reverse transcriptase activity protocol; WMPC, white matter progenitor cells. 


\subsection{Locations investigated regarding possible adult human neurogenesis}

Tables 1 and 3 and Figure 3 detail the locations and number of specimens investigated with in vivo or in vitro methods. Overall, the most explored zones were the temporal and extra-temporal SVZ and the hippocampus. Less explored zones included the temporal, parietal, and frontal cortices, white matter, amygdala, septum, striatum, and corpus callosum.

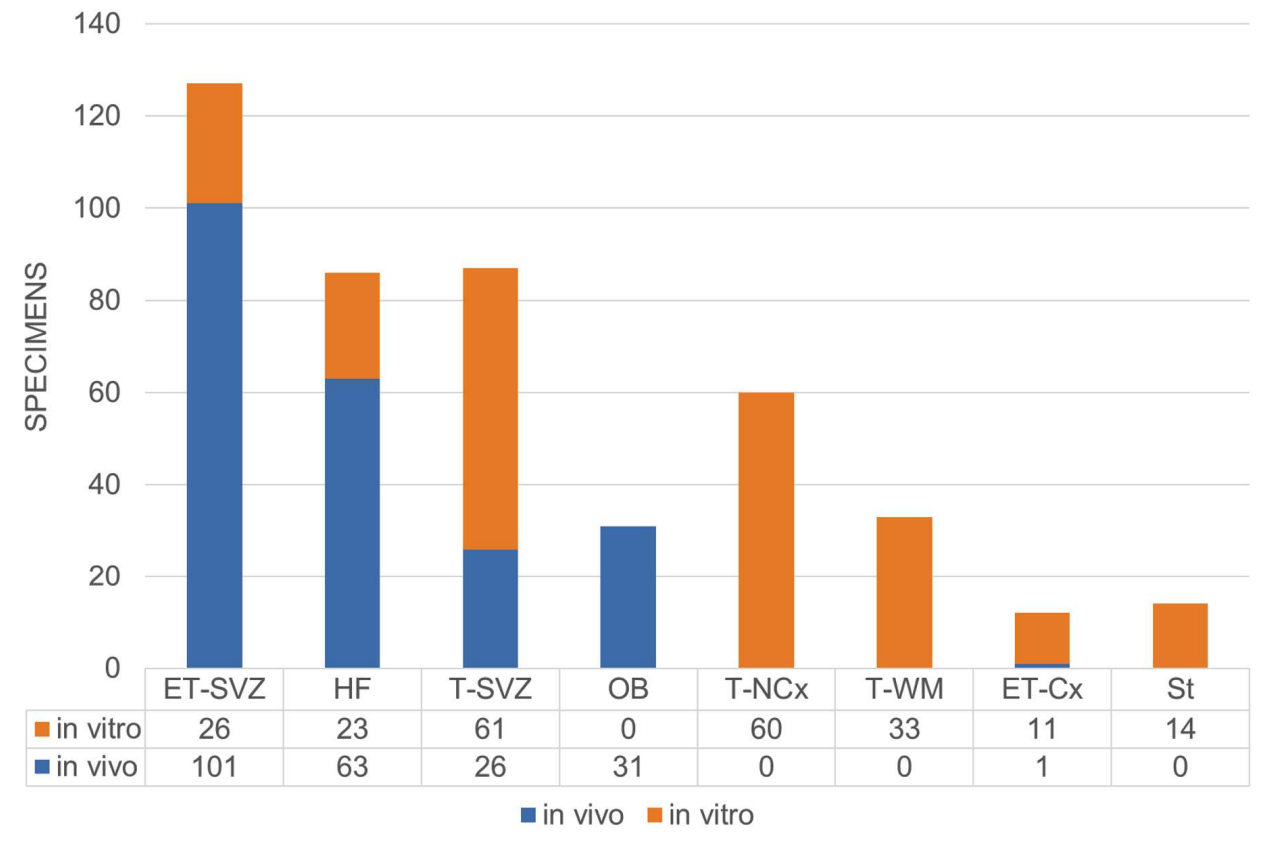

Figure 3. Number of specimens and locations analyzed to detect adult human neurogenesis in manuscripts published up to 2004. Most of the studies focused on the search of evidence for human neurogenesis in known mammalian neurogenic niches, represented in the first four bars. The last four bars reflect principally the fact that the availability of surgical samples for cell culture guided the chosen for other locations to be studied. One specimen of the corpus callosum analyzed by in vitro method is not represented in this graph. Blue and orange bars represent the number of specimens analyzed by in vivo and in vitro methods, respectively. Legend: Cx, cortex; ET, extra-temporal; HF, hippocampal formation; $\mathrm{NCx}$, neocortex; OB, olfactory bulb; SVZ, subventricular zone; St, striatum; T, temporal; WM, white matter.

\subsection{Results of the original articles on adult human neurogenesis}

\subsubsection{In vitro methods showed putative NSCs principally in the SVZ and hippocampus}

All studies with samples from the hippocampus (seven manuscripts) (Johansson et al. 1999; Kukekov et al. 1999; Roy et al. 2000a; Roy et al. 2000b; Akiyama et al. 2001; Arsenijevic et al. 2001; Palmer et al. 2001) and from the SVZ (ten manuscripts (Kirschenbaum et al. 1994; Pincus et al. 1997; Johansson et al. 1999; Kukekov et al. 1999; Roy et al. 2000a; Akiyama et al. 2001; Arsenijevic et al. 2001; Palmer et al. 2001; Westerlund et al. 2003; Sanai et al. 2004), mostly analyzing the temporal SVZ (Pincus et al. 1997; Johansson et al. 1999; Kukekov et al. 1999; Roy et al. 2000a; Akiyama et al. 2001; Arsenijevic et al. 2001; Westerlund et al. 2003)) were able to characterize NSCs 
in vitro. Samples from the white matter (Palmer et al. 2001; Nunes et al. 2003) and the temporal (Eriksson et al. 1998; Pincus et al. 1998; Arsenijevic et al. 2001; Palmer et al. 2001) and frontal (Arsenijevic et al. 2001; Palmer et al. 2001) cortices contained NSCs, although some laboratories did not replicate these findings regarding the white matter (Kirschenbaum et al. 1994) and temporal (Kirschenbaum et al. 1994; Kukekov et al. 1999; Akiyama et al. 2001), frontal (Sanai et al. 2004), and parietal (Sanai et al. 2004) cortices. Neurogenesis capacity was detected in the amygdala (Arsenijevic et al. 2001), olfactory bulb (Pagano et al. 2000), and corpus callosum (Palmer et al. 2001), in one study for each of these locations. Further information regarding the percentage of cells in the culture medium that corresponded to adult human NSCs, the proliferation capacity of these cells, the phenotypes found in adult human NSC culture, the influence of growth factors added to the culture media, and the contribution of other methods to detect and characterize adult human NSCs are detailed in Tables $7-9$. The results achieved with in vitro methods suggest that the adult human brain harbors NSCs in the two canonical neurogenic niches and possibly in other cortical and subcortical locations (Table 10).

Table 7. Results (in vivo + retrospective in vitro methods)
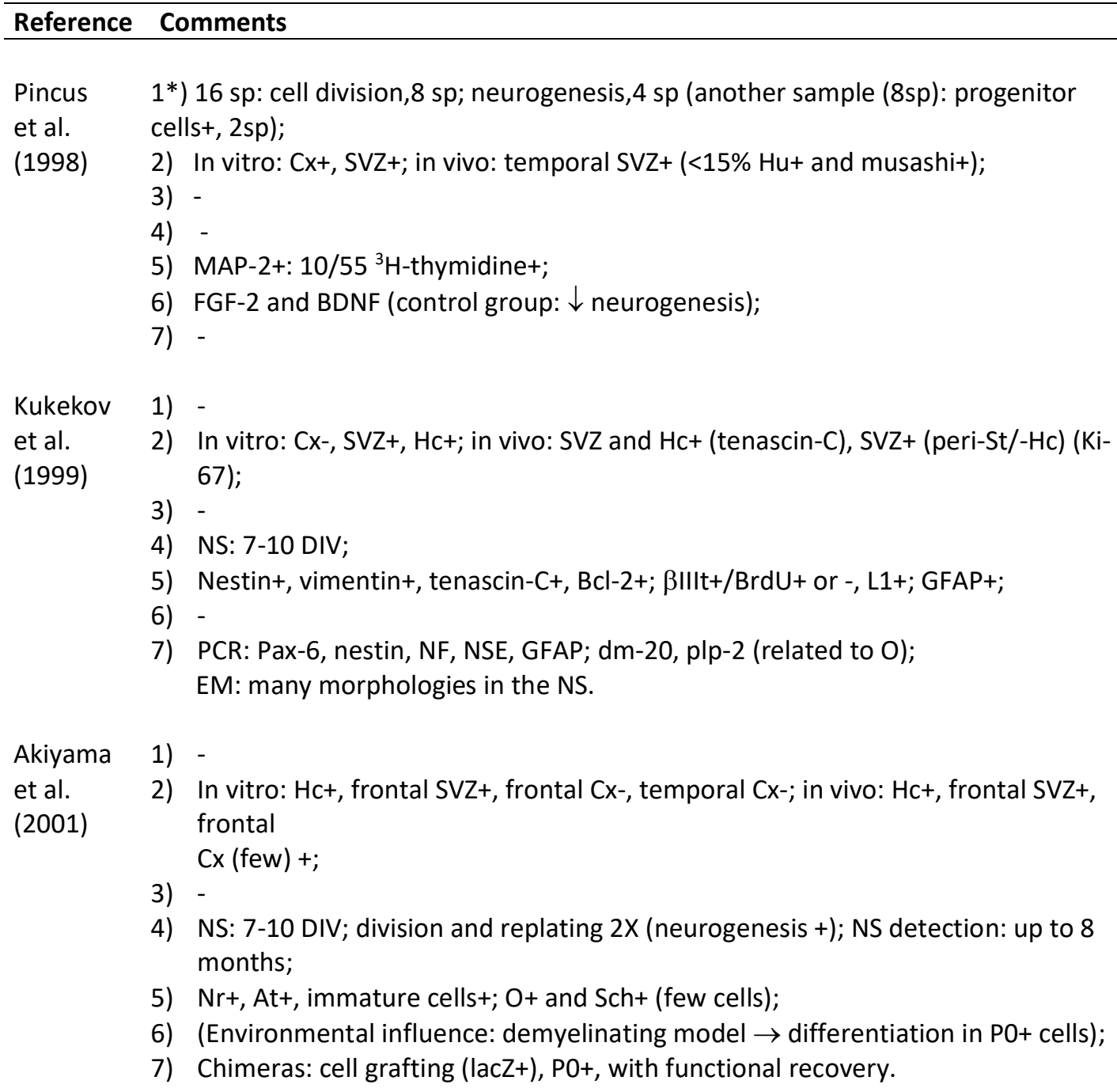
et al. (2004)
2) In vitro: SVZ+ (lateral ventricles); $C x$ (parietal, temporal)-, St-, septum-; in vivo: SVZ+ (lateral ventricles - astrocyte ribbon: $0.77 \pm 0.29 \%$ GFAP+/Ki-67+); SVZ (III and IV ventricles)-, septum-; no evidence for RMS (PSA-NCAM- and $\beta$ IIIt- cells);

3) Some GFAP+ and BrdU+ (in microdissected SVZ); $62.57 \pm 7.46 \mathrm{NS} / \mathrm{ml}$ (with 50,000 cells $/ \mathrm{ml}$ ); $109.29 \pm 8.76 \mathrm{NS} / \mathrm{ml}$ (with 50,000 SVZ At/ml); 2.9-4.1\% SVZ green At** genereted clonal NS, Cx and near-SVZ At did not;

4) $33.11 \pm 3.81$ secondary NS / 100 primary NS; SVZ At grew at higer rate than cortical At;

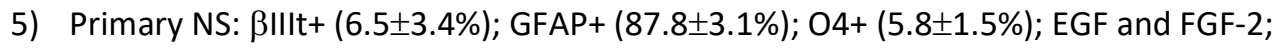
(SVZ At onto human cortical At monolayer in serum free medium generated $\beta \mathrm{IIIt+}$ cells (cortical At did not));

6) EM: At in SVZ.

*Questions (Tables 7 and 8): 1) what was the percentage of specimens that generated adult human NSCs?; 2) what was the location of adult human NSCs in the brain?; 3) what percentage of the cultured cells corresponded to adult human NSCs?; 4) how was the proliferation ability of adult human NSCs?; 5) what were the phenotypes found in adult human NSC cultures? 6) what was the influence of growth factors? 7) what was the contribution of other methods for understanding of the adult human NSCs potential?; ** astrocytes expressing GFP after incubation for 3 days with adenovirus carrying the gene for green fluorescent protein controlled by the GFAP promoter; At, astrocyte; Bcl-2, anti-apoptotic protein Bcl-2; BDNF, brain derived neurotrophic factor; $B$ rdU, bromodeoxyuridine; $C X$, cortex; DIV, days in vitro; $E M$, electron microscopy; FGF-2, basic fibroblast growth factor; GFAP, glial fibrillary acid protein; GFAPp, green fluorescent protein controlled by GFAP promoter; Hc, hippocampus; lac-Z, $\beta$ galactosidase; L1, adhesion molecule L1; MAP, microtubule-associated protein; NF, neurofilament; Nr, neuron; NS, neurosphere (formation); NSE, neuron specific enolase; $\mathrm{O}$, oligodendrocyte; PCR, polymerase chain reaction; PSA-NCAM, polysialylated form of neural cell adhesion molecule; RMS, rostral migratory stream; Sch, Schwann cells; SVZ, subventricular zone; sp, specimens; St, striatum; $\beta$ IIIt, $\beta$ III-tubulin.

Table 8. Results (retrospective in vitro methods)

Reference Comments

Kirschenbaum $\left.1^{*}\right) 11 \mathrm{sp}$ : 6 sets of cultures; neurogenesis: $3 \mathrm{sp}$;

et al. $\quad$ 2) Temporal SVZ+, CX-, WM-, (gliogenesis: SVZ+, $C_{x+}, W M+$ );

(1994) 3) $2 \times 10^{5}$ cells: $79 \mathrm{GFAP}+{ }^{3} \mathrm{H}$-thymidine+; $6 \mathrm{MAP}-2 /{ }^{3} \mathrm{H}$-thymidine+;

4) 14 DIV;

5) $393 \mathrm{GFAP}+: 20 \%{ }^{3} \mathrm{H}$-thymidine+; 56 neural markers+: $11 \%{ }^{3} \mathrm{H}$-thymidine+; $\mathrm{O} 4+$ : $\downarrow S V Z, \uparrow W M$;

6) EGF;

7) Calcium imaging: $\uparrow \uparrow \mathrm{Ca}^{2+}(\mathrm{Nr}), \uparrow \mathrm{Ca}^{2+}(\mathrm{At})$.

Pincus 1) Neurogenesis: $1 / 8 \mathrm{sp}$ (total author's cases (27 sp): 2 cortical neurogenesis);

et al. 2) Temporal $\mathrm{Cx}+$;

(1997) 3) -

4) 9 weeks (period of observation);

5) MAP-2: $20 \%{ }^{3} \mathrm{H}$-thymidine+;

6) FGF-2, BDNF;

7) Calcium imaging: MAP-2+: $\uparrow \uparrow \mathrm{Ca}^{2+}$.

Johansson 1) -

et al. $\quad$ 2) $\mathrm{Hc}+$, temporal SVZ+; 
(1999)

3) -

4) NS: 2 weeks; division and replating, 1x; (4 NS / 168 SVZ cells; 2 NS / 40 Hc cells);

5) $\mathrm{Nr}+, \mathrm{At}+, \mathrm{O}+$;

6) EGF, FGF-2;

7) -

Pagano 1)

et al. $\quad$ 2) Olfactory bulb+;

(2000) 3) $1 \%$;

4) Cell division: 3 DIV; cell proliferation: up to 20 passages;

5) (7 DIV / 3 passages): $\mathrm{Nr}+: 16.21 \pm 1.49 \%, \mathrm{At}+: 65.8 \pm 2.6 \%, 04+: 2.1 \pm 0.96 \%$;

6) EGF, FGF-2, (LIF-1: $\uparrow \% \mathrm{Nr})$;

7) -

Arsenijevic 1) -

et al. $\quad$ 2) $\mathrm{Hc}+, \mathrm{Amy}_{+}$, temporal SVZ and $\mathrm{CX}_{+}$, frontal $\mathrm{CX}^{+}$;

(2001) 3) $10^{6}$ cells: $8-1449 \mathrm{NS}$;

4) NS: division and replating: new NS up to 3-4 passages; cell proliferation: 2 weeks; NS: 1 month;

5) $\uparrow \uparrow \beta$ IIIt,$+ \uparrow$ MAP-2+, $\uparrow$ N-CAM, $\varnothing$ Neu-N; $11 \%$ neural marker+ and GFAP or $\mathrm{O} 4+$; O4+; $143 \mathrm{Nr} / \mathrm{NS}$ (+BDNF);

6) EGF, FGF-2, BDNF, PDGF; (EGF + FGF-2: $\uparrow \uparrow$ cells; no growth factors: $\varnothing$ cells);

7) Clonal origin: lac- $Z$ and $\beta$ IIIt or GFAP expression; PCR.

Palmer 1) -

et al. $\quad$ 2) Post-mortem: $\mathrm{Hc}+, \mathrm{SVZ}+$, motor $\mathrm{Cx}+, \mathrm{CC}+$; epilepsy: $\mathrm{Cx}+, \mathrm{WM}+, \mathrm{Hc}+$;

(2001) 3) > number of NS: Hc and SVZ;

4) Population doubling before senescence: post-mortem: 70; epilepsy: 30;

5) $\mathrm{Nr}+, \mathrm{At}+, \mathrm{O}+$;

6) EGF, FGF-2, PDGF $+25 \%$ conditioned medium from rat stem cells, modified to overproduce a form of FGF-2 and the glycosylated form of cystatin C; 7) -

Westlund 1) -

et al. $\quad$ 2) Temporal SVZ+;

(2003) 3) -

4) NS formation: 2-3 weeks; after 3-7 weeks: NS with a few hundred cells; NS division and replating ( 2 passages); cells kept in culture for up to 11 months;

5) 7 days after a second passage: $63 \% \mathrm{GFAP}+, 14 \% \mathrm{O} 4+, 23 \% \beta \mathrm{Illt}$; NeuN+ cells only after the $7^{\text {th }}$ day;

6) EGF, FGF-2;

7) Patch-clamp: recordings at different developmental stages: Nr-like cells behavior is in keeping with observations made in developing brain.

* Questions presented in Table 7; Amy, amygdala; At, astrocyte; BDNF, brain derived neurotrophic factor; $\mathrm{CC}$, corpus callosum; $\mathrm{Cx}$, cortex; DIV, days in vitro; EGF, epithelial growth factor; FGF-2, basic fibroblast growth factor; GFAP, glial fibrillary acid protein; Hc, hippocampus; LIF-1, leukemia inhibitory factor; MAP, microtubule-associated protein; N-CAM, neural cell adhesion molecule; Neu-N, neuron-specific nuclear protein; $\mathrm{Nr}$, neuron; NS, neurospheres (formation); O, oligodendrocyte; PCR, polymerase chain reaction; PDGF, platelet 
derived growth factor; SVZ, subventricular zone; sp, specimens; WM, white matter; $\beta$ IIIt, $\beta$ IIItubulin.

Table 9. Results (prospective (+retrospective in vitro) methods)

\begin{tabular}{|c|c|c|c|c|}
\hline Reference & $\begin{array}{l}\text { Neurogenesis } \\
\text { (location) } \\
+ \text { or - }\end{array}$ & $\begin{array}{l}\% \text { adult } \\
\text { human NSCs* }\end{array}$ & Phenotypes** / Proliferation capacity & $\begin{array}{l}\text { Growth } \\
\text { Factors }\end{array}$ \\
\hline $\begin{array}{l}\text { Roy et al. } \\
\text { (2000b) }\end{array}$ & $\begin{array}{l}\text { Dentate } \\
\text { gyrus+ }\end{array}$ & $\begin{array}{l}0.53 \pm 0.20 \% \\
\text { E/nestin:EGFP } \\
+, \\
(62.5 \pm 2.9 \% \\
\beta I I I t+, \\
\text { some GFAP+); } \\
1.11 \% \pm 0.35 \% \\
\text { P/T } 1 \text { 1:hGFP+, } \\
(73.2 \pm 6.6 \% \\
\beta I I I t+)\end{array}$ & 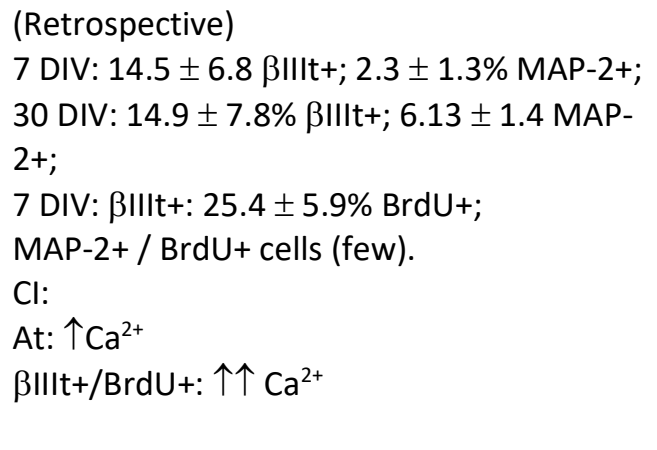 & $\begin{array}{l}\text { FGF-2, } \\
\text { BDNF }\end{array}$ \\
\hline
\end{tabular}

\begin{tabular}{|c|c|c|c|c|}
\hline $\begin{array}{l}\text { Roy et al. } \\
(2000 a)\end{array}$ & $\begin{array}{l}\text { Temporal } \\
\text { SVZ+ }\end{array}$ & $\begin{array}{l}0.11 \pm 0.2 \% \\
\text { E/nestin:EGFP } \\
+, \\
\text { (3 patients); } \\
2719 \text { SVZ cells: } \\
0.92 \pm 0.2 \% \\
\text { P/T } \alpha 1: \text { hGFP+, } \\
(76 \pm 7.6 \% \\
\beta I I I t+)\end{array}$ & $\begin{array}{l}7 \text { DIV: } 562 \beta \text { IIIt+ cells: } 104 \text { BrdU+. } \\
\text { E/nestin:EGFP+ / BrdU+ / } \beta \text { Illtt+ and/or } \\
\text { MAP- } 2+\text { cells }\end{array}$ & FGF-2 \\
\hline $\begin{array}{l}\text { Nunes et } \\
\text { al. (2003) }\end{array}$ & $\begin{array}{l}\text { Temporal } \\
\text { WM+ }\end{array}$ & 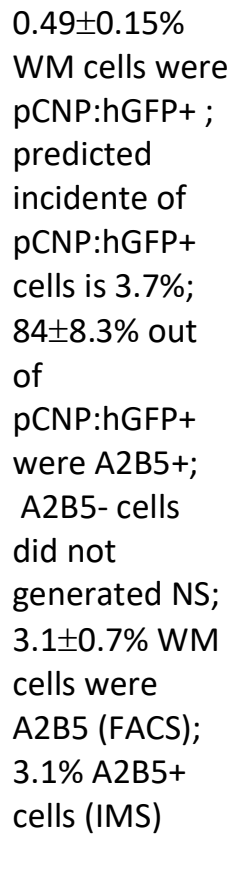 & $\begin{array}{l}\text { NS after } 17 \text { days; } 3 \text { weeks after sorting: } \\
84.8 \pm 9.0 \text { spheres/well; } 46.5 \pm 8.2 \\
\text { cells/sphere; secondary NS: } 2 \text { weeks after } \\
\text { plating primary NS cells (with O4+, GFAP+, } \\
\text { and } \beta \text { IIIt+ cells); NS achieved up to terciary } \\
\text { NS, no quaternary NS; } \\
\text { 50,000-100,000 cells/ml } \rightarrow \text { best density for } \\
\text { expansion of WMPC; } \\
\text { NS: estimated 16-24 - } 30 \text { doublings; } \\
\text { BrdU+/another marker (O4, GFAP, } \beta \text { IIIt, Hu } \\
\text { protein, or MAP-2)+ cells; } \\
\text { Proportion Nr (e.g. gabaergic):At:O } \rightarrow \\
\text { primary secondary NS; } \\
\text { No telomerase activity; } \\
\text { Cl and patch-clamp: physiological } \\
\text { responses expected for Nr and At; WMPC- } \\
\text { derived neurons generated fast sodium } \\
\text { currents and action potentials; } \\
\text { Clonal origin: each NS with GFP+ or - cells } \\
\text { (after lentiviral GFP labeling); }\end{array}$ & $\begin{array}{l}\text { FGF-2, } \\
\text { PDGF, } \\
\text { NT-3; } \\
\text { No } \\
\text { reprog. } \\
\text { steps } \\
\text { were } \\
\text { required } \\
\text { for } \\
\text { neuro } \\
\text { genesis }\end{array}$ \\
\hline
\end{tabular}


Chimera: $\mathrm{HNA}+$ /nestin+ or DCX+ or $\beta$ IIIt+ cells in olfactory subependyma, $\mathrm{Hc}$, and neoSt (HNA+/ $\beta I I t+$ cells in RMS); human WMPC-derived GFAP+ At and CNP+ O in the ventricles or in the subcortical WM *\% of adult human NSCs / specimen (both in culture and/or in brain sections); ** determined by immunostaining (including in vitro generated cells), or other methods; At, astrocytes; BDNF, brain derived neurotrophic factor; $\mathrm{BrdU}$, bromodeoxyuridine; $\mathrm{Cl}$, calcium imaging; $\mathrm{CMV}$, citomegalovirus; DCX, doublecortin; DIV, days in vitro; (E)GFP, enhanced GFP; E/nestin, nestin enhancer; FGF-2, basic fibroblast growth factor; GFAP, glial fibrillary acid protein; Hc, hippocampus; hGFP, humanized green fluorescent protein; IMS, immunomagnetic sorting; MAP, microtubule-associated protein; NS, neurospheres (formation); pCNP2, promoter for CNP (early oligodendrocytic transcript); PDGF, platelet-derived growth factor; P/T $\alpha 1, T \alpha 1$ tubulin promoter; RMS, rostral migratory stream; SVZ, subventricular zone; St, striatum; WM, white matter; WMPC, white matter progenitor cells; $\beta$ IIIt, $\beta$ III-tubulin.

Table 10. Results (in vitro neurogenesis and brain structures)

Structure

$\begin{array}{ll}\text { Hippocampus } & 7 * \\ \text { Temporal subventricular zone } & 8 \\ \text { Temporal white matter } & 2 \\ \text { Temporal cortex } & 4 \\ \text { Frontal subventricular zone } & 2 \\ \text { Frontal cortex } & 2 \\ \text { Parietal cortex } & \\ \text { Striatum } & 1 \\ \text { Olfactory bulb } & \\ \text { Subventricular zone } & \\ \text { Subventricular zone-septum } & \\ \text { Corpus callosum } & \\ \text { *number of papers. } & \end{array}$

$7 *$

8

$2 \quad 1$

$4 \quad 4$

2

21

1

1

1

2

1

\subsubsection{Location of possible adult human neurogenesis assessed by in vivo methods}

\section{Presence of staining of neurogenesis markers in the SGZ}

Mitosis activity and the presence of NSCs were demonstrated in the hippocampus (Table 11). A heterogeneous neurogenesis in different hippocampus zones was demonstrated in a study that examined the autopsy brains of patients submitted to bromodeoxyuridine (BrdU) injection for detection of newly formed cells as part of cancer management (Eriksson et al. 1998). BrdU+ cells were present, in decreasing order, in the subgranular and granular layer, and in the hilus. Generally, there was a colocalization of BrdU with neuronal markers and sometimes with glial markers (Eriksson et al. 1998). 
Table 11. Results (In vivo methods)

Reference Neurogenesis (location) + or - Phenotypes

Eriksson et al. Number of BrdU+ cells: subgranular (1998) zone $(D G)>$ granule cell layer (DG)> hilus;

SVZ+ (adjacent to the caudate nucleus)

Bernier et al.

(2000) of the anterior comissure, ventral
SVZ+ (nestin+ cells, mainly at the level striatum, and septum)

Blümcke et al. Hc+ (epileptic patients ( $>2$ years-old), (2001)

Curtis et al. (2003)

Liu and Martin (2003)

Bédard and

$\mathrm{OB}+$

Parent (2004) few nestin+ cells in the molecular layer and hilus); Hc- (non-epileptic patients and postnatal autopsies)

SVZ+ adjacent to $\mathrm{CN}$

$\mathrm{OB}+$ (cell density: younger > elderly individuals)
BrdU+ cells: Neu-N+, 22\%; NSE+, 22,7\%;

Calbindin+, 7,9\%; GFAP+ 18,1\%

Nestin + / GFAP+ (many); nestin + / PCNA+ $(\downarrow)$; PSA-NCAM+ / $\beta I I I t+; B c l-2+$

(Significant immunoreactivity: only in epileptic children ( $<2$ years-old))

PCNA+ (Huntington's disease $>$ control); few TUNEL+ cells in the SVZ; $\beta$ IIIt+ or $\mathrm{NeuN}+\mathrm{SVZ}$ adjacent to CN; GFAP+ or vimentin+ SVZ and in the $\mathrm{CN}$; PCNA+/ $\beta \mathrm{IIIt+}$ ( $5 \%$ out of PCNA+ cells); PCNA+/GFAP+ ( $50 \%$ out of PCNA+ cells)

GFAP+ (always TUC4 -); $\mathrm{Neu}-\mathrm{N}+$ (some TUC4+); $\beta \mathrm{ll} \mathrm{lt}+$; Nestin+ / vimentin+ (posterior end of the $\mathrm{OB})$

Ki-67+, PCNA+ (GIL, GrL); $\beta$ IIIt+/DCX+, and nestin+/PCNA+ (GrL); Ki-67+ and NeuroD+ (GIL); GAD+ (GIL, GrL); TH+ (GIL); $\beta$ IIIt+ and $\mathrm{CR}+, \mathrm{TH}+$, or $\mathrm{PV}+$ (periGIL); distribution of PSA-NCAM+ and DCX+ cells RMS;

Western blot: expression of PCNA

Jim et al. $\quad \mathrm{Hc}+$

(2004) (AD > controls)

Halmarks of AD (A $\beta$, APP, phospho- $\tau$ )+; Controls and AD: TUC-4+, DCX+, cleavage product of caspase-8+ (SGZ);

Only in AD: TUC-4+ (GCL), DCX+ (GCL and CA1);

Co-marcation in AD: TUC-4+/Hu+ cells, $\mathrm{DCX}+$ / PSA-NCAM+ cells; 
Western blot: $\uparrow$ DCX, PSA-NCAM, TUC-4 in AD patients; (calbindin and NeuN not incresead)

$A D$, Alzheimer's disease; APP, amyloid precursor protein; $A \beta, \beta$ amyloid peptide; $B c l-2$, antiapoptotic protein $\mathrm{Bcl}-2$; $\mathrm{BrdU}$, bromodeoxyuridine; $\mathrm{CN}$, caudate nucleus; $\mathrm{CR}$, calretinin; $\mathrm{DCX}$, doublecortin; DG, dentate gyrus; GAD, glutamic acid decarboxylase; $G C L$, granule cell layer; GFAP, glial fibrillary acid protein; GIL, glomerular layer; GrL, granular layer; Hc, hippocampus; Neu-N, neuron-specific nuclear protein; NSE, neuron specific enolase; OB, olfactory bulb; PCNA, proliferating cell nuclear antigen; PSA-NCAM, polysialylated form of neural cell adhesion molecule; PV, parvalbumin; RMS, rostral migratory stream; SVZ, subventricular zone; SGZ, subgranular zone; TH, tyrosine hydroxylase; TUC-4, turned on / Unc-33-like phosphoprotein-1 / CRMP-4 family; TUNEL, terminal deoxynucleotidyltransferase-mediated dATP nick and labeling; $\beta$ IIIt, $\beta$ III-tubulin.

Jin et al. (2004) (Table 11) studied the brain of normal individuals and Alzheimer's disease patients. In both cases, cells with early neuronal markers were found in the subgranular layer. However, only the hippocampus of Alzheimer's disease patients had stained cells in the granular cell layer and in the CA1, demonstrating more significant neurogenesis in these cases. Furthermore, Western Blot analysis revealed that the hippocampus of Alzheimer's disease patients in comparison to controls displayed more significant expression of immature neuron markers (Jin et al. 2004).

\section{Minimal staining of neurogenesis markers in the SGZ}

Blümcke et al. (2001) carried out another study using nestin as NSC marker, suggesting the existence of NSCs at the hippocampus mostly in the molecular layer and in the hilus (Table 11). Nonetheless, only the hippocampus of epileptic children younger than two years of age presented a significant number of nestin+ cells. Non-epileptic adult hippocampi obtained in autopsies did not stained for nestin.

\section{SVZ and RMS}

Diffuse staining of neurogenesis markers in the SVZ of structures of the limbic system, hypothalamus, and basal ganglia

The entire SVZ was immunoreactive to the neurogenesis-related markers applied by Bernier et al. (2001) (Table 11) including the hypothalamic portion of the third ventricle. The thickest immunoreactive area in the SVZ was near the anterior commissure and the ventral striatum. The septum also displayed significant immunostaining for neurogenesis markers.

\section{Staining of neurogenesis markers in restricted parts of the SVZ}

Sanai et al. (2004) found cell division markers in the SVZ of the lateral ventricles, in vivo (Table 11). These authors calculated that $0.77 \pm 0.29 \%$ of glial fibrillary acidic protein (GFAP)+ NSCs in the SVZ were in division (Ki-67+). On the other hand, the same authors did not detect cell division in the SVZ near the septum or surrounding the III and IV ventricles.

\section{Contradictory results on the existence of the RMS}

Bédard and Parent (2004) found distribution of cells expressing the immature neuronal markers polysialylated-neural cell adhesion molecule (PSA-NCAM) and doublecortin (DCX) from the olfactory peduncle to the olfactory bulb (Table 11). On the other hand, 
Sanai et al. (2004) did not find cells marked with immature neuronal markers (PSANCAM and BIII-tubulin), between the SVZ at the floor of the anterior horn of the lateral ventricle and the olfactory trigone (the origin of the olfactory peduncle) (Table 11), suggesting that the RMS does not exist in humans.

\section{Second-era studies: persistence of contradictory results on the existence of RMS}

Broadly, second-era studies corroborated that the SGZ is a neurogenic niche in humans (Nogueira et al. 2014b). Nonetheless, the most remarkable debate during this period involved manuscripts assessing the existence of the RMS in humans (Curtis et al. 2007; Sanai et al. 2011; Wang et al. 2011). The conclusions were either that the RMS exists only during a short period of the infancy in a trajectory different from the expected (Sanai et al. 2011) or that the RMS exists in adults in a trajectory different from rodents (Curtis et al. 2007; Wang et al. 2011). We discuss below a possible explanation for this contradiction with basis on our results (Nogueira et al. 2014b), which showed a pattern in which the expression of neurogenesis markers follows neural circuits instead of consisting of a separate zone embedded into the parenchyma.

\section{Third-era studies: contradictory results on SGZ as a neurogenic niche}

The lack of signs of neurogenesis at the SGZ showed by Sorrells et al. (2018) triggered a new debate on this issue (Table 12). Nonetheless, three other studies showed indications of neurogenesis at the SGZ that remained across aging (Boldrini et al. 2018) and declined in mild cognitive impairment (and not in Alzheimer's disease) (Tobin et al. 2019) or declined in Alzheimer's disease (Moreno-Jimenéz et al. 2019).

Table 12. Main features of histologically normal human brains in recent papers on human subgranular zone neurogenesis

\begin{tabular}{|c|c|c|c|}
\hline & $\begin{array}{l}\text { Premortem agonal } \\
\text { period }\end{array}$ & $\begin{array}{l}\text { Time elapsed } \\
\text { between death and } \\
\text { tissue fixation }\end{array}$ & $\begin{array}{l}\text { Larger } \\
\text { cytoarchitecture } \\
\text { zones shown in } \\
\text { published images }\end{array}$ \\
\hline Sorrells et al. (2018) & Non-specified & $\begin{array}{l}<48 \mathrm{~h}, \text { two samples } 3 \\
-5 \mathrm{~h}\end{array}$ & $\begin{array}{l}\text { Small microscopic } \\
\text { fields involving } \\
\text { molecular layers, } \\
\text { granule cell layer, } \\
\text { and SGZ }\end{array}$ \\
\hline Boldrini et al. (2018) & Non-specified & $4-26 h$ & $\begin{array}{l}\text { One image with } \\
\text { molecular layers, } \\
\text { granule cell layer, } \\
\text { SGZ, and hilus }\end{array}$ \\
\hline $\begin{array}{l}\text { Moreno-Jiménez et } \\
\text { al. (2019) }\end{array}$ & Non-specified & $\begin{array}{l}2.5-10 \mathrm{~h} \text {, three } \\
\text { samples with } \\
\text { postmortem delay of } \\
16,23, \text { and } 38 \mathrm{~h} \\
\text { (total } 58 \text { subjects } \\
\text { analyzed in this } \\
\text { study, } 13 \\
\text { neurologically } \\
\text { healthy subjects) }\end{array}$ & $\begin{array}{l}\text { One image showing } \\
\text { the hippocampal } \\
\text { formation } \\
\text { (subiculum not } \\
\text { analyzed; CA-1 } \\
\text { analyzed for DCX } \\
\text { expression but not } \\
\text { nestin) }\end{array}$ \\
\hline Tobin et al. (2019) & Non-specified & $\begin{array}{l}4.92-43.55 \mathrm{~h} \text { (mean } \\
10.28 \mathrm{~h})\end{array}$ & $\begin{array}{l}\text { One image showing } \\
\text { internal molecular }\end{array}$ \\
\hline
\end{tabular}


CA, Cornu Ammonis; DCX, doublecortin; SGZ, subgranular zone.

layer, granule cell

layer, SGZ and its

boundary with CA4

\section{Discussion}

\subsection{Experimental and theoretical factors may have led to different results and interpretations of results on adult human neurogenesis}

\subsubsection{Human brain cytoarchitecture: the necessity to encompass the zone from the choroid plexus to the subiculum in SGZ studies}

We put forth that the explanation for contradictory results regarding SGZ neurogenesis in the third-era studies is that the SGZ is not the primary neurogenic niche in the human hippocampal formation (Nogueira et al. 2014b; Nogueira et al. 2018). Although the negative results by Sorrells et al. (2018) are the opposite of the results of the vast majority of investigations on neurogenesis at the human SGZ, they are not unprecedented. The first-era article by Blümcke et al. (2001) contains high-quality images of nestin staining in children SGZ that was practically absent in normal adults.

Our results are a midterm among the results of the third-era manuscripts because they revealed that human SGZ neurogenesis exists but is a less significant part of a larger zone expressing neurogenesis markers (Figure 1) (Nogueira et al. 2014b; Nogueira et al. 2018). In the temporal lobe, this zone begins at the choroid plexus ependymal cell layer (which expresses the NSC marker CD133) (Nogueira et al. 2014b), encompasses nestin+ cells with long processes at the taenia fimbria, follows the subpial zone of the medial temporal lobe, bifurcates as a minor branch toward the SGZ and a major branch toward the CA1. At the CA1, the expression of nestin ends up and gives rise to cells expressing DCX adjacently, at the subiculum. Our findings suggest that the conundrum of the human SGZ neurogenesis will not be clarified with studies that disregard the analysis of the medial temporal lobe as a whole.

The analysis of the temporal lobe we put forth implies the demonstration of the cytoarchitecture structures expressing neurogenesis markers and, as precise as possible, the site where samples obtained for in vitro experiments were harvested (Nogueira et al. 2014b; Nogueira et al. 2017c; Nogueira et al. 2018). For example, on the one hand the harvesting of cells exclusive from the dentate gyrus (Roy et al. 2000b) is unlikely because of microneurosurgical issues (Wen et al. 1999). On the other hand, Jonas Frisén's team (Spalding et al. 2013) published an article (together with comments by Gerd Kempermann (2013)) that for some reason described only vaguely where in a large structure such as the human temporal lobe they sought indications of neurogenesis.

\subsubsection{Influence of tissue fixation delay and agonal period has not been sufficiently addressed}

The second most important factor for contradictory results on human neurogenesis is probably the time elapsed between death and tissue fixation. For example, we showed that nestin + cells are detected when the time elapsed between death and tissue fixation is less than 16 hours on average (Nogueira et al. 2014b). Sorrells et al. (2018) fixed brains after a time span larger than 16 hours and obtained no neurogenesis marker 
staining at the SGZ (although they also have not found this staining in hippocampus samples fixed immediately after surgical resection in epilepsy cases).

Agonal period is rarely specified in articles on adult human neurogenesis. None of the third-era studies mentioned this period (Boldrini et al. 2018; Sorrels et al. 2018; Moreno-Jiménez et al. 2019; Tobin et al. 2019). We specified the agonal period of individuals whose brains were studied as a whole (Nogueira et al. 2014b), which should be mandatory in studies with negative results: long agonal period may lead for example to the "respirator brain", with degradation of neurogenesis-related proteins.

\subsubsection{Theoretical assumption that neurogenesis must be more significant in rodents than in humans could influence the interpretation of results}

The dogma of "no neuron formation after birth" has been eliminated but it seems that it was replaced by the dogma stating that adult human neurogenesis must be proportionally less significant than adult mammalian neurogenesis in general. A curious fact that may be underneath this unmentioned assumption is the publication in a paper regarded seminal of an image of a hippocampus that would be displayed correctly for rodents but is inverted in the craniocaudal direction because it indeed shows a human hippocampus (Eriksson et al. 1998). This disposition perhaps indicates an analysis of results influenced by the familiarity of the authors with rodent brain neurogenesis and anatomy. In other words, perhaps researchers in the field of adult mammalian neurogenesis assume a priori that experiments showing staining of neurogenesis markers in humans proportionally more significant than in rodents are likely a technical error.

Contrarywise, Arsenijevic et al. (2001) and Bernier et al. (2000) found indications that adult human neurogenesis may be not restricted to two discrete and separate neurogenic niches. Likewise, we showed indications that hippocampal formation neurogenesis may have been shifted from the SGZ-granule cell layer zone in rodents to the CA1subiculum zone in humans (Nogueira et al. 2014b; Nogueira et al. 2018). This idea is coherent with the fact that the cortical structure where the three-layer cortex becomes the six-layer cortex, i.e. the subiculum, displays one of the major increases in primate brain structures in comparison to rodents (Nogueira et al. 2014b; Nogueira et al. 2018).

\subsection{Recent studies brought no novelty on location of adult human neurogenesis and disagree on neurogenesis in Alzheimer's disease}

Three recent studies replicated findings of neurogenesis in the SGZ (Table 12) (Boldrini et al. 2018; Moreno-Jimenéz et al. 2019; Tobin et al. 2019). However, their findings disagree between each other and between a first-era study regarding Alzheimer's disease (Jin et al. 2004). Neurogenesis in Alzheimer's disease was increased in the firstera study (Jin et al. 2004), normal in a third-era study but with diminishment in mild cognitive impairment (Tobin et al. 2019) and decreased in another study (MorenoJimenéz et al (2019). Moreover, aging led to a decrease in brain plasticity and angiogenesis although neurogenesis remained at the same level in one of these studies (Boldrini et al. 2018). Therefore, the behavior of the SGZ as a neurogenic niche in Alzheimer's disease remains to be determined. 


\subsection{Current prevailing approach of adult human neurogenesis studies may rekindle possible misinterpretations on human RMS}

Our previous findings (Nogueira et al. 2014b) lead to the prediction that if the lack of investigation of expression of neurogenesis markers beyond the boundaries of canonical neurogenic niches is repeated in further studies on the SVZ and RMS, than the probable result will be the return of controversies on the RMS because of the same technical aspects that rekindled controversies on the human SGZ (Nogueira et al. 2014b; Nogueira et al. 2017c; Nogueira et al. 2018).

Previous studies concluded that the human RMS does not exist (Sanai et al. 2004) or exists in a different trajectory in comparison to other mammals during a short post-natal period (Sanai et al. 2011) or during adulthood (Curtis et al. 2007; Wang et al. 2011). We detailed somewhere else (Nogueira et al. 2014b) that the analysis of a larger brain zone allowed the conclusion that the likely explanation for this controversy is that the zone thought to be the human RMS is indeed the column of the fornix expressing neurogenesis markers (Nogueira et al. 2014b).

Alternatively, the most recent second-era paper assessing the human RMS performed analysis in a more anterior zone and published high-quality images with large brain sections (Wang et al. 2011). In this case, the zone analyzed may be related to the neurogenesis marker staining at the anterior hypothalamus or septal area (Nogueira et al. 2014b; Wang et al. 2011).

\subsection{Bigger picture analysis may enhance alternative conclusions of an orchestrated adult human neurogenesis}

This review showed that recent studies (Boldrini et al. 2018; Sorrels et al. 2018; Moreno-Jiménez et al. 2019; Tobin et al. 2019) reported no novelty on location of neurogenic niches at the human temporal lobe. Moreover, some of these recent studies did not take advantage of previous experimental issues involving premortem agonal state and time elapsed between death and tissue fixation (Nogueira et al. 2014b). Only one study published larger hippocampal formation image with identification of the dentate gyrus, hippocampus proper, and surrounding structures (Moreno-Jimenéz et al. 2019). However, this study did not search for nestin staining at CA1 or DCX staining adjacently at the subiculum (Moreno-Jimenéz et al. 2019).

Remarkably, the possibility of existence of neurogenesis in a broad brain area was identified by Bernier et al. (2000) in a first-era study that remains practically unnoticed. These authors showed anti-apoptotic and NSC markers distributed across the SVZ of the limbic system, hypothalamus, and ventral striatum (Bernier et al. 2000) Moreover, these results were reinforced by findings of the same team in non-human primates (Bernier and Parent 1998), which also displayed neurogenesis in a broad area in another study (Gould et al. 1999) that was received with criticism by the time of its publication (Rakic 2002).

Our previous findings follow the line of evidence revealed principally by Bernier et al. (2000), together with in vitro indications of neurogenesis in non-canonical neurogenic niches such as the amygdala (Arsenijevic et al. 2001). We added novel structures displaying staining of neurogenesis markers in such a way that they seem to reveal a potential neurogenic system that encompasses the SGZ and SVZ (Figures 1 and 2) 
(Nogueira et al. 2014b; Nogueira et al. 2017c; Nogueira et al. 2018). In short, the potential neurogenic system begins at the circumventricular organs, which are the structures without blood-brain barrier, and follows sequentially across the hypothalamus and limbic system, with gradual vanishing of staining of neurogenesis markers reaching structures of the reticular activating system (Nogueira et al. 2014b).

A caveat on the existence of a neurogenic system is that there is no explanation for its mechanisms. Joseph Altman's indications of adult mammalian neurogenesis was thought to be unlikely because neurons display no figure of mitosis (Altman 1962). The discovery that the adult mammalian brain harbors NSCs that form new neurons solved this issue. Perhaps neurogenesis could also involve neuron DNA replication and the formation of heterokaryons (Giordano-Santini et al. 2016) (curiously, Altman showed an image of binucleated cortical neurons of rats with at least one nucleus stained for newly synthesized DNA) (Altman 1963). Moreover, our identification of a massive DCX staining in trajectories of certain neural circuits disproportional to the expected neurogenesis rate may have identified neurons in a "stand-by" mode (Marichal et al. 2009). Furthermore, NSCs transplanted into a model of spinal cord injury displayed axonal growth through a long distance (Lu et al. 2012). In short, mechanisms of a potential adult human neurogenic system can only be imagined for now and the methods to assess them remain to be developed.

\subsection{A hypothesis: neuronal cells formed before or after birth express respectively fetal or adult hemoglobin}

\subsubsection{Lack of specific marker of newly formed neuron}

The lack of a specific marker that proves that a neuron is newly formed or formed in the post-natal period is another major limitation in adult human neurogenesis studies (Nogueira et al. 2014b). Markers currently used to identify newly formed neurons are expressed in neurons generated in the fetus that remained immature after birth (Piumatti et al. 2018; Sorrells et al. 2019; La Rosa et al. 2020a; La Rosa et al. 2020b; Seki 2020) or re-expressed in neurons as a response to conditions such as aging and inflammation (Hagihara et al. 2019).

Nonetheless, as we have previously discussed (Nogueira et al. 2014b), one possible explanation for the detection of expression of neurogenesis-related markers in the adult human brain is that it is indeed identifying cells involved in post-natal neurogenesis, such as neural stem cells and newly formed neurons. Accordingly, this possible explanation and its caveats are normally mentioned and taken into consideration in the original studies analyzed in this review. Anyway, the findings of these original studies could serve as basis to figure out the puzzle on locations of adult human neurogenesis, principally when a method that proves the existence of adult human neurogenesis will be revealed.

\subsubsection{Location of expression of putative neurogenesis markers may contribute to the revelation of adult human neurogenesis}

The locations where cells express different markers of the process of neurogenesis do not prove but are indications compatible with the existence of adult human neurogenesis. For example, hypothalamic neurogenesis is controversial in mammals in general. However, our results showing the expression of nestin in the circumventricular 
organs (which may be reached by systemic, cerebrospinal fluid, and neural factors) (Nogueira et al. 2014b), surrounded by DCX-expressing brain nuclei, indicate that hypothalamic neurogenesis is at least plausible in adult humans.

Furthermore, our demonstration that the status of hypothalamus-related functions may predict the occurrence of neurologic signs (Kim et al. 2015; Nogueira and Loddenkemper 2017; Nogueira et al. 2017a; Nogueira et al. 2017b) is coherent with a possible existence of an endogenous mechanism of brain plasticity, perhaps involving neurogenesis. In this regard, we showed that the pattern of circadian oscillation of temperature correlates with the likelihood of occurrence in the following 24 hours of seizure in epileptic patients (Kim et al. 2015; Nogueira and Loddenkemper 2017) and of intracranial hypertension in cases of severe stroke (Nogueira et al. 2017a; Nogueira et al. 2017b). We speculate that normal hypothalamus-related functions clinically detectable reflect a normal hypothalamic plasticity and neurogenesis that serve as an endogenous protection against the installation of neurologic signs (Nogueira and Loddenkemper 2017; Nogueira et al. 2017a; Nogueira et al. 2017b).

\subsubsection{Hypothesis that a panel of expression of globins could determine time of birth and degree of maturity of neural cells}

We hypothesize that the lack of prove of adult human neurogenesis can be overcome using a panel of globins (Schelshorn et al. 2009; Haines et al. 2013; Emara et al. 2014). Our approach would begin with the analysis of the expression of the relatively recently described neuroglobin as a marker of immature neuron (Haines et al. 2013). Second, to detect whether an immature neuron was born before or after birth we propose to carry out immuno-histochemistry experiments to reveal hemoglobin staining in neurons (Schelshorn et al. 2009; Emara et al. 2014). We wonder whether the expression of fetal hemoglobin shifts to the expression of adult hemoglobin in neurons in the same way it occurs in red blood cells. The rationale for this idea is that the expression of adult hemoglobin in adult neurons has already been shown (Schelshorn et al. 2009). To the best of our knowledge, the expression of fetal hemoglobin in fetal neurons has not been studied, however, such expression would follow the same physiological pattern of red blood cells; furthermore, it has already been shown that glioblastoma samples, which contains glioblastoma stem cells, display expression of fetal hemoglobin (Emara et al. 2014).

The primary expected result would be the co-expression of neuroglobin and adult hemoglobin in immature neurons formed after birth. Importantly, this hypothesis could be validated using, for example, the BrdU paradigm in rodents. The results could be reproduced easily, especially in comparison to other methods used to detect the formation of neurons in the adult human brain that in some researchers' view actually may be detecting DNA repair instead of DNA replication during the formation of a neuron (Sorrells et al. 2018).

\section{Conclusion}

We conclude that the prevailing view on adult human neurogenesis has been developed by minor increments as from the period when the "two-neurogenic-niche concept" in humans was consolidated. We propose that the standardization of minimal requirements for research on adult human neurogenesis could foster research in this field (Nogueira et al. 2014b; Nogueira et al. 2017c; Nogueira et al. 2018). We suggest that further studies should bear in mind the possibility that adult human neurogenesis is organized across 
the brain circuitry, involves the brain as a whole (Nogueira et al. 2014b; Nogueira et al. 2017c; Nogueira et al. 2018) and is a primary factor of brain plasticity (Nogueira et al., 2014a; Nogueira et al. 2014b; Kim et al. 2015; Nogueira and Loddenkemper 2017; Nogueira et al. 2017a; Nogueira et al. 2017c).

\section{Acknowledgements}

The authors thank the collaborators of their original research on adult human neurogenesis.

\section{Conflict of interest}

The authors declare that they have no conflict of interest.

\section{Availability of data and material}

This manuscript does not contain original data.

\section{Author contributions}

ABN: wrote the manuscript; HRSH: reviewed the manuscript; BGSS: reviewed the manuscript; NCO: reviewed the manuscript; MJT: reviewed the manuscript.

\section{Ethics approval}

This is a review paper on existing studies. No original data has been used in this manuscript.

\section{Funding: None}

\section{References}

Akiyama Y, Honmou O, Kato T, Uede T, Hashi K, Kocsis JD (2001) Transplantation of clonal neural precursor cells derived from adult human brain establishes functional peripheral myelin in the rat spinal cord. Exp Neurol 167:27-39. https://doi.org/10.1006/exnr.2000.7539

Altman J (1962) Are new neurons formed in the brains of adult mammals? Science 135:1127-1128. https://doi.org/10.1126/science.135.3509.1127

Altman J (1963) Autoradiographic investigation of cell proliferation in the brains of rats and cats. Anat Rec 145:573-591. https://doi.org/10.10002/ar.1091450409

Arsenijevic Y, Villemure JG, Brunet JF et al (2001) Isolation of multipotent precursors residing in the cortex of the adult human brain. Exp Neurol 170:48-62. https://doi.org/10.1006/exnr.2001.7691

Bédard A, Parent A (2004) Evidence of newly generated neurons in the human olfactory bulb. Brain Res Dev Brain Res 151:159-168. https://doi.org/10.1016/j.devbrainres.2004.03.021

Bernier PJ, Parent A (1998) Bcl-2 protein as a marker of neuronal immaturity in postnatal primate brain. J Neurosci 18:2486-2497. https://doi.org/10.1523/JNEUROSCI.18-07-02486.1998

Bernier PJ, Vinet J, Cossette M, Parent A (2000) Characterization of the subventricular zone of the adult human brain: evidence for involvement of Bcl-2. Neurosci Res 37:67-78. https://doi.org/10.1016/S01680102(00)00102-4 
Blümcke I, Schewe JC, Normann S et al (2001) Increase in nestin-immunoreactive neural precursor cells in the dentate gyrus of pediatric patients with early-onset temporal lobe epilepsy. Hippocampus 11:311321. https://doi.org/10.1002/hipo.1045

Boldrini M, Fulmore CA, Tartt NA et al (2018) Human hippocampal neurogenesis persists throughout aging. Cell Stem Cell 22:589-599. https://doi.org/10.1016/j.stem.2018.03.015

Curtis MA, Penney EB, Pearson AG et al (2003) Increased cell proliferation and neurogenesis in the adult human Huntington's disease brain. Proc Natl Acad Sci USA 100:9023-9027.

https://doi.org/10.1073/pnas. 1532244100

Curtis MA, Kam M, Nannmark U et al (2007) Human neuroblasts migrate to the olfactory bulb via a lateral ventricular extension. Science 315:1243-1249. https://doi.org/10.1126/science.1136281

Emara M, Turner AR, Allalunis-Turner J (2014) Adult, embryonic and fetal hemoglobin are expressed in human glioblastoma cells. Int J Oncol 44:514-520. https://doi.org/10.3892/ijo.2013.2186

Eriksson PS, Perfilieva E, Björk-Eriksson T et al (1998) Neurogenesis in the adult human hippocampus. Nat Med 4:1313-1317. https://doi.org/10.1038/3305

Giordano-Santini R, Linton C, Hilliard MA (2016) Cell-cell fusion in the nervous system: Alternative mechanisms of development, injury, and repair. Semin Cel Dev Biol 60:146-154.

https://doi.org/10.1016/j.semcdb.2016.06.019

Gould E, Reeves AJ, Graziano MS, Gross CG (1999) Neurogenesis in the neocortex of adult primates. Science 286:548-552. https://doi.org/10.1126/science.286.5439.548

Hagihara H, Murano T, Ohira K, Miwa M, Nakamura K, Miyakawa T (2019) Expression of progenitor cell/immature neuron markers does not present definitive evidence for adult neurogenesis. Mol Brain. https://doi.org/10.1186/s13041-019-0522-8

Haines B, Mao X, Xie L, Spusta S, Zeng X, Jin K, Greenberg DA (2013) Neuroglobin expression in neurogenesis. Neurosci Lett 549:3-6. https://doi.org/10.1016/j.neulet.2013.04.039

Jin K, Peel AL, Mao XO et al (2004) Increased hippocampal neurogenesis in Alzheimer's disease. Proc Natl Acad Sci USA 101:343-347. https://doi.org/10.1073/pnas.2634794100

Johansson CB, Svensson M, Wallstedt L, Janson AM, Frisén J (1999) Neural stem cells in the adult human brain. Exp Cell Res 253:733-736. https://doi.org/10.1006/excr.1999.4678

Kempermann G (2013) Neuroscience. What the bomb said about the brain. Science 340:1180-1181. https://doi.org/10.1126/science. 1240681

Kim B, Nogueira AB, Thome-Souza S et al (2015) Diurnal and nocturnal patterns of autonomic neurophysiological measurements are related to timing of seizures. Annals of Neurol 78(S19):S174-175

Kirschenbaum B, Nedergaard M, Preuss A, Barami K, Fraser RA, Goldman SA (1994) In vitro neuronal production and differentiation by precursor cells derived from the adult human forebrain. Cereb Cortex 4:576-589. https://doi.org/10.1093/cercor/4.6.576

Kukekov VG, Laywell ED, Suslov O et al (1999) Multipotent stem/progenitor cells with similar properties arise from two neurogenic regions of the adult human brain. Exp Neurol 156:333-344. https://doi.org/10.1006/exnr.1999.7028

Kumar A, Pareek V, Faig MA, Ghosh SK, Kumari C (2019) Adult neurogenesis in humans: a review of basic concepts, history, current research, and clinical implications. Innov Clin Neurosci 16:30-37

La Rosa C, Cavallo F, Pecora A et al (2020a) Phylogenetic variation in cortical layer II immature neuron reservoir of mammals. eLife. https://doi.org/10.7554/eLife.55456

La Rosa C, Parolisi R, Bonfanti L (2020b) Brain structural plasticity: from adult neurogenesis to immature neurons. Front Neurosci. https://doi.org/10.3389/fnins.2020.00075

Liu Z, Martin LJ (2003) Olfactory bulb core is a rich source of neural progenitor and stem cells in adult rodent and human. J Comp Neurol 459:368-391. https://doi.org/10.1002/cne.10664 
Lu P, Wang Y, Graham L et al (2012) Long-distance growth and connectivity of neural stem cells after severe spinal cord injury. Cell 150:1264-1273. http://doi.org/10.1016/j.cell.2012.08.020

Ming GL, Song H (2011) Adult neurogenesis in the mammalian brain: significant answers and significant questions. Neuron 70:687-702. https://doi.org/10.1016/j.neuron.2011.05.001

Marichal N, García G, Radmilovich M, Trujillo-Cenóz O, Russo RE (2009) Enigmatic central canal contacting cells: immature neurons in "standby mode"? J Neurosci 29:10010-10024.

https://doi.org/10.1523/JNEUROSCI.6183-08.2009

Moreno-Jiménez EP, Flor-Garcia M, Terreros-Roncal J et al (2019) Adult hippocampal neurogenesis is abundant in neurologically healthy subjects and drops sharply in patients with Alzheimer's disease. Nat Med 25:554-560. https://doi.org/10.1038/s41591-019-0375-9

Nogueira AB, Nogueira AB, Veiga JCE, Teixeira MJ (2014a) Multimodality monitoring, inflammation, and neuroregeneration in subarachnoid hemorrhage. Neurosurgery 75:678-689.

https://doi.org/10.1227/NEU.0000000000000512

Nogueira AB, Sogayar MC, Colquhoun A et al (2014b) Existence of a potential neurogenic system in the adult human brain. J Transl Med. https://doi.org/10.1186/1479-5876-12-75

Nogueira AB, Loddenkemper T (inventors), Boston Children's Hospital (applicant) (2017) Seizure prediction based on comparison of biological information across wake and sleep periods. World Intellectual Property Organization (WIPO). Int Class A61B 5/00. Appl No. PCT/US2016/041085. Publication date: January 12, 2017

Nogueira AB, Annen E, Boss O, Farokhzad F, Sikorski F, Keller E (2017a) Temperature variability in the day-night cycle is associated with further intracranial pressure during therapeutic hypothermia. $\mathrm{J}$ Transl Med https://doi.org10.1186/s12967-017-1272-y

Nogueira AB, Nogueira AB, Veiga JCE, Teixeira MJ (2017b) Hypothesis on the role of cryptochromes in inflammation and subarachnoid hemorrhage outcome. Front Neurol.

https://doi.org/10.3389/fneur.2017.00637

Nogueira AB, Nogueira AB, Veiga JCE, Teixeira MJ (2017c) Letter: Extensive migration of young neurons into the infant human frontal lobe. Neurosurgery. https://doi.org/10.1093/neuros/nyx202

Nogueira AB, Nogueira AB, Veiga JCE, Teixeira MJ (2018) Letter: Human hippocampal neurogenesis drops sharply in children to undetectable levels in adults. Neurosurgery.

https://doi.org/10.1093/neuros/nyy252

Nunes MC, Roy NS, Keyoung HM et al (2003) Identification and isolation of multipotent neural progenitor cells from the subcortical white matter of the adult human brain. Nat Med 9:439-447. https://doi.org/10.1038/nm837

Pagano SF, Inpagnatiello F, Girelli M et al (2000) Isolation and characterization of neural stem cells from the adult human olfactory bulb. Stem Cells 18:295-300. https://doi.org/10.1634/stemcells.18-4-295

Palmer TD, Schwartz PH, Taupin P, Kaspar B, Stein SA, Gage FH (2001) Progenitor cells from human brain after death. Nature 411:42-43. https://doi.org/10.1038/35075141

Pincus DW, Harrison-Restelli C, Barry J et al (1997) In vitro neurogenesis by adult human epileptic temporal neocortex. Clin Neurosurg 44:17-25

Pincus DW, Keyoung HM, Harrison-Restelli C et al (1998) Fibroblast growth factor-2/brain-derived neurotrophic factor-associated maturation of new neurons generated from adult human subependymal cells. Ann Neurol 43:576-585. https://doi.org/10.1002/ana.410430505

Piumatti M, Palazzo O, La Rosa C et al (2018) Non-newly generated, "immature" neurons in the sheep brain are not restricted to cerebral cortex. J Neurosci 38:826-842. https://doi.org/10.1523/JNEUROSCI.1781-17.2017

Rakic P (2002) Neurogenesis in adult primate cortex: an evaluation of the evidence. Nat Rev Neurosci 3:65-71. https://doi.org/10.1038/nrn700 
Roy NS, Benraiss A, Wang S et al (2000a) Promoter-targeted selection and isolation of neural progenitor cells from the adult human ventricular zone. J Neurosci Res 59:321-331. https://doi.org/10.1002/(sici)1097-4547(20000201)59:3<321::aid-jnr5>3.0.co;2-9.

Roy NS, Wang S, Jiang L et al (2000b) In vitro neurogenesis by progenitor cells isolated from the adult human hippocampus. Nat Med 6:271-277. https://doi.org/10.1038/73119

Sanai N, Tramontin AD, Quiñones-Hinojosa A et al (2004) Unique astrocyte ribbon in adult human brain contains neural stem cells but lacks chain migration. Nature 427:740-744. https://doi.org/10.1038/nature02301

Sanai N, Nguyen T, Ihrie RA et al (2011) Corridors of migrating neurons in the human brain and their decline during infancy. Nature 478:382-386. https://doi.org/10.1038/nature10487

Schelshorn DW, Schneider A, Kuschinsky W et al (2009) Expression of hemoglobin in rodent neurons. J Cereb Blood Flow Metab 29:585-595. https://doi.org/10.1038/jcbfm.2008.152

Seki T (2020) Understanding the real state of human adult hippocampal neurogenesis from studies of rodents and non-human primates. Front Neurosci. https://doi.org/10.3389/fnins.2020.00839

Sorrells SF, Paredes MF, Cebrian-Silla A et al (2018) Human hippocampal neurogenesis drops sharply in children to undetectable levels in adults. Nature 555:377-381. https://doi.org/10.1038/nature25975

Sorrells SF, Paredes MF, Velmeshev D (2019) Immature excitatory neurons develop during adolescence in the human amygdala. Nat Commun. https://doi.org/10.1038/s41467-019-10765-1

Spalding KL, Bergmann O, Alkass K et al (2013) Dynamics of hippocampal neurogenesis in adult humans. Cell 153:1219-1227. https://doi.org/10.1016/j.cell.2013.05.002

Tobin MK, Musaraca K, Disouky A et al. (2019) Human hippocampal neurogenesis persists in aged adults and Alzheimer's disease patients. Cell Stem Cell 24:974-982.

https://doi.org/10.1016/j.stem.2019.05.003

Wang C, Liu F, Liu YY et al (2011) Identification and characterization of neuroblasts in the subventricular zone and rostral migratory stream of the adult human brain. Cell Res 21:1534-1550. https://doi.org/10.1038/cr.2011.83

Wen HT, Rhoton AL, de Oliveira E et al (1999) Microsurgical anatomy of the temporal lobe: part 1: mesial temporal lobe anatomy and its vascular relationships as applied to amygdalohippocampectomy. Neurosurgery 45:549-591. https://doi.org/10.1097/00006123-199909000-00028

Westerlund U, Moe MC, Varghese M et al (2003) Stem cells from the adult human brain develop into functional neurons in culture. Exp Cell Res 289:378-383. https://doi.org/10.1016/S0014-4827(03)00291$\mathrm{X}$ 